\title{
Singular Adventures of Baron Bourgain in the Labyrinth of the Continuum
}

Alexander Gamburd
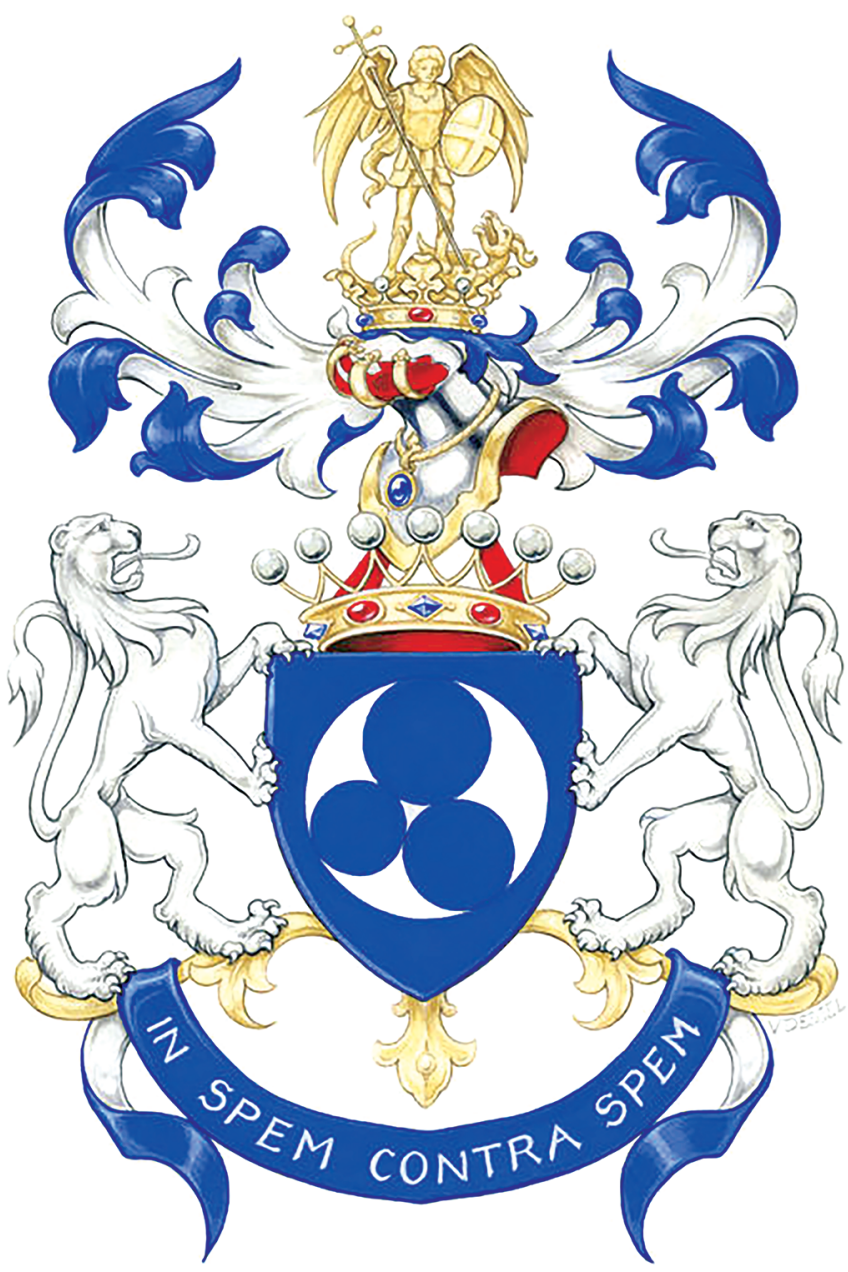

Alexander Gamburd is Presidential Professor of Mathematics at the Graduate Center, CUNY. His email address is agamburd@gmai 1 . com.

A coat of arms of Jean Bourgain, who was bestowed the title of Baron by King Philippe of Belgium in July 2015.

Communicated by Notices Associate Editor Daniela De Silva.

For permission to reprint this article, please contact:

reprint-permission@ams .org.

DOI: https://doi.org/10.1090/noti2191
There are two labyrinths of the human mind: one concerns the composition of the continuum, and the other the nature of freedom, and both spring from the same source-the infinite.

\section{Baron von Leibniz}

During World War II, when von Neumann was working on the design of nuclear weapons, he came to the conclusion that analytical methods were inadequate to the task, and that the only way to deal with equations of continuum mechanics is to discretize them. ... It is to this task that von Neumann devoted his energies after the war.

Peter Lax

\section{Overture}

Baron Bourgain, the IBM von Neumann Professor in the School of Mathematics at the Institute for Advanced Study (IAS), was one of the most original, penetrating and versatile analytical minds of our troubled times, justly celebrated $^{1}$ and revered without reservations.

While he rejected outright the suggestion of a sixtieth birthday conference, a proposal to have a gathering occasioned by the publication of his 500th paper was not immediately dismissed-the conference Analysis and Beyond: Celebrating Jean Bourgain's Work and its Impact took place at the IAS in Princeton on May 21-24, 2016. The conference talks (all of which were videotaped) are a tribute to the depth and breadth of Bourgain's work and its singular and transcendent impact on the whole of our discipline. The beauty and power of the first result highlighted by Jean's hand on the conference poster $\left\|e^{i t \Delta} \varphi\right\|_{p} \ll N^{\varepsilon}\|\varphi\|_{q}$ is apparent from reading the splendid paper by Andrea

${ }^{1}$ An excerpt from Bourgain's interview upon receiving the 2017 Breakthrough Prize in Mathematical Sciences concludes this essay.

The following quote is from The Work of Jean Bourgain by Luis Caffarelli, Proceedings of ICM, 1994 (the year Bourgain was awarded the Fields Medal): 'Bourgain's work touches on several central topics of mathematical analysis: the geometry of Banach spaces, convexity in high dimensions, harmonic analysis, ergodic theory, and, finally, nonlinear partial differential equations from mathematical physics. In all of these areas, he made spectacular inroads into questions where progress has been blocked for a long time. This he did by simultaneously bringing into play different areas of mathematics: number theory, combinatorics, probability, and showing their relevance to the problem in the previously unforeseen fashion.' 
Nahmod in the Bulletin of the American Mathematical Society (BAMS), 2016. The brief of this paper is to explicate the origins, nature and development of the second result, the discretized sum-product inequality

$$
\mathcal{N}(A+A, \delta)+\mathcal{N}(A \cdot A, \delta)>\mathcal{N}(A, \delta)^{1+\tau},
$$

in Analysis and beyond.

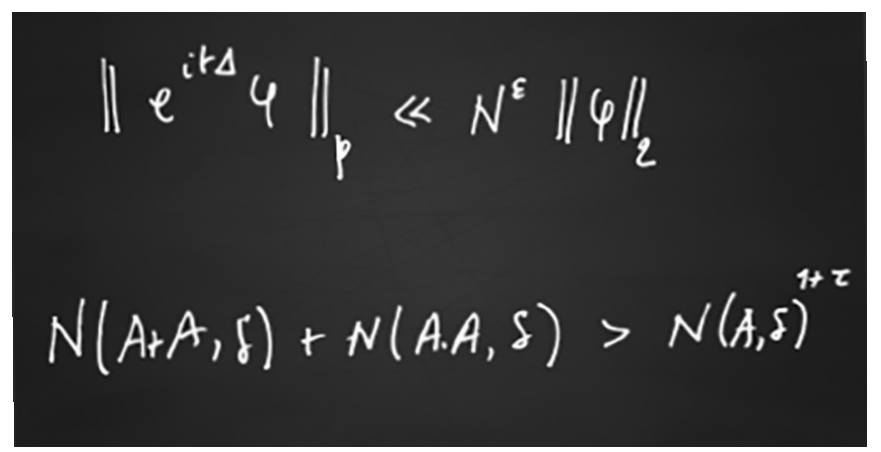

Figure 1. Two of Jean Bourgain's signature results.

The three great branches of mathematics are, in historical order, Geometry, Algebra and Analysis. Geometry we owe essentially to Greek civilization, Algebra is of Indo-Arab origin and Analysis (or Calculus) was the creation of Newton and Leibniz, ushering in the modern era.

Sir Michael Atiyah

Von Zahlen und Figuren-'On Numbers and Shapes' ${ }^{2}$-is the title of one of the most successful expositions of mathematics aimed at a broad audience, reflecting a common perception of our discipline as a marriage between Algebra and Geometry. This happy marriage, notwithstanding Count Tolstoy's contention ('All happy marriages are alike; each unhappy marriage is unhappy in its own way.'), is not without tensions (as, perhaps, each happy marriageincluding, possibly, bicameral mind-is in its own way). 'In these days the angel of topology and the devil of abstract algebra fight for the soul of each individual mathematical domain' is the way Hermann Weyl ${ }^{3}$ put it; three score and seven years later, in a conversation at Google with the company's CEO, a somewhat divergent sentiment was expressed: 'When you form your ideas on the basis of words, you build from concepts, which to be meaningful depend on relation to other concepts. When you form your ideas on the basis of pictures, you form your views on the basis of impressions and of moods, that cannot even

\footnotetext{
${ }^{2}$ The book was written in 1933 by Hans Rademacher and Otto Toeplitz, two outstanding analysts of the past century, who made a deliberate decision not to refer in their exposition to the Analysis (or Calculus) of Leibniz and Newton. The English translation is entitled The Enjoyment of Math.

${ }^{3}$ In Invariants, Duke Mathematics Journal 5 1939, anticipating by four years an even more sweeping assertion, due to Jean-Paul Sartre: 'L'enfer, c'est les autres.'
}

be recreated very easily, so you cannot look back and check what it was that impressed you so much.'

This tension is embodied in the system of real numbers, the soil in which the functions of Analysis grow, resembling Janus's head facing in two directions: on the one hand it is the field closed under the operations of addition and multiplication; on the other hand, it is a continuous manifold the parts of which are so connected as to defy exact isolation from each other. The one is algebraic, the other is the geometric face of real numbers. Continued fractions is a much more intrinsic and geometric form of discretizing the continuum; the lack of a practical algorithm for their addition and multiplication leads to the regnancy of the discretization based on the ordinary (digital or decimal, i.e., base 10) fractions.

Whereas Newton, in his development of Calculus, was primarily motivated by 'dynamics' (force, acceleration), as exemplified by the falling of the apple on his head, Leibniz, it appears, was more intrigued by what would now be described by the appellation 'fractal geometry of nature.' 'Imagine a circle; inscribe within it three other circles congruent to each other and of maximum radius; proceed similarly within each of these circles and within each interval between them, and imagine that the process continues ad infinitum, wrote Leibniz referencing a configuration akin to the four mutually tangent circles appearing on Baron Bourgain's coat of arms. Leibniz's definition of the straight line as a 'curve, any part of which is similar to the whole, and it alone has this property, not only among curves but among sets' is a reflection of the fractal nature of the continuum: the Cantor set would satisfy Leibniz's definition. ${ }^{5}$

Dynamics, broadly conceived, is perceived as a study of change, which in its primordial (physical) context takes place within time. The Cantor set (and $\mathbb{R}$ ) are, so to speak, static in time, but there is 'a condition of possibility' of (almost) 'equi-primordial' change 'in the eye of the beholder', taking form in changing the degree of magnification scale and 'zooming in.' This is reflected in the 'multi-scale' nature of Bourgain's proof(s) of (1).

To bring this opening section to a close, let us in passing note that both results chosen by Jean are not equalities (inequalities, rather), commenting thus:

If Algebra is generally perceived as the study of equations, what perhaps lies at the heart of Analysis are inequalities, or estimates, which compare the size of two quantities or expressions. Einstein's discovery that nothing

\footnotetext{
${ }^{4}$ Henry Kissinger.

${ }^{5}$ Leibniz also wrote the first textbook on combinatorics, Dissertatio de arte combinatoria, and invented the binary notation, which made possible modern computers and will play an important role in navigating the labyrinth of Bourgain's argument.

The first collection of Leibniz's works was published in 1735 by Rudolf Erich Raspe, better known today for his authorship of Singular Adventures of Baron Munchausen.
} 
travels faster than light is an example of an inequality. The inequality $2^{X}$ is considerably larger than $X$ arguably neatly encapsulates both the P vs NP problem (properly stated for finite $X$ ) and Cantor's Continuum Problem (when $X$ is the first unfinite ordinal). An elementary inequality, taught in the middle school, asserts that the arithmetic mean of two positive numbers is never less than their geometric mean. In between these two extremes there is a vast range of estimates of great variety and importance. Such estimates, reflecting and quantifying some subtle aspect of the underlying problem, are often exceedingly difficult to prove. It will be seen that for the inequality (1), with which we are about to get intimate, the underlying issue lies at the heart of the tension between the algebraic and (fractal)geometric nature of the continuum. Fractal derives from Latin fractus, meaning broken apart; algebra, derives from the Arabic al-jabr, meaning the reunion of broken parts.

\section{Origins: Kakeya-Besicovitch Problem+}

\begin{abstract}
It is difficult and often impossible to judge the value of a problem correctly in advance; for the final award depends upon the gain which science obtains from the problem. Nevertheless we can ask whether there are general criteria which mark a good mathematical problem. An old French mathematician said: 'A mathematical theory is not to be considered complete until you have made it so clear that you can explain it to the first man whom you meet on the street.' This clearness and ease of comprehension, here insisted on for a mathematical theory, I should still more demand for a mathematical problem if it is to be perfect; for what is clear and easily comprehended attracts, the complicated repels us.
\end{abstract}

David Hilbert, Problems of Mathematics, 1900

Hilbert's ${ }^{6}$ democratic dictum, if followed by Sōichi Kakeya (writing the paper on an island nation in 1917, at the height of the Great War) the explanation of the problem now bearing his name to almost every person at just about any street in Eastern Eurasia might have run as follows: Entrusted with defending an island, possessing a huge hill, cragged and steep, your task is to purchase at the least cost to the nation's treasury, a plot of land on the flat hilltop with the following property: a cannon of length one must be capable of pointing in any direction.

Kakeya improved by a factor of one-half the obvious solution (a circle of diameter one, having area $\frac{\pi}{4}$ ); his proposed shape (three-cusped hypocycloid inscribed in the circle of radius 1 ) is alluded to in the rendering of $A$ in the conference poster (Figure 2). In the same year, working in Perm, ${ }^{7}$ while the October/November Russian/Soviet

\footnotetext{
${ }^{6}$ Hilbert's paper on Dirichlet's Principle is one of the two referenced by Kakeya; the second one (also on Dirichlet's principle) is by Caratheodory, a student of Hilbert. In his magnificent book Geometry and Imagination Hilbert refers to Besicovitch's result (described below) as 'showing that this [Kakeya] problem has no solution.'

${ }^{7}$ Subsequently Molotov (1940-1957); currently Perm.
}

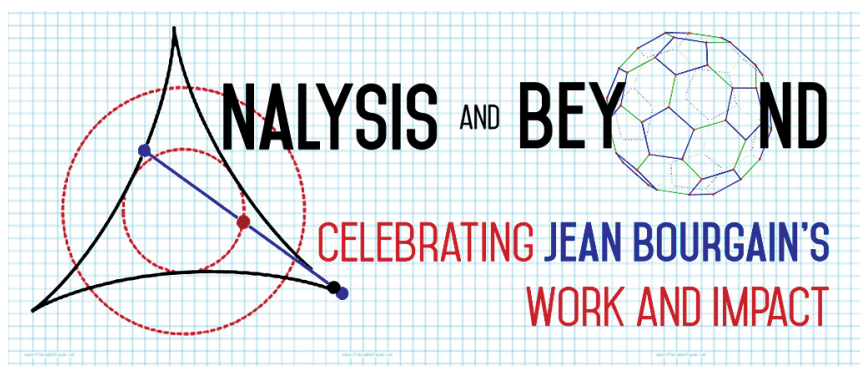

Figure 2. Analysis and Beyond.

Revolution was unfolding, A. S. Besicovitch reduced the minimal necessary sum to virtually ${ }^{8}$ nothing.

In fact, Besicovitch was working on the following question: if $f$ is a Riemann integrable function defined on the plane, is it always possible to find a pair of orthogonal coordinate axes with respect to which $\int f(x, y) d x$ exists as a Riemann integral for all $y$, and with the resulting function of $y$ also Riemann integrable? Besicovitch noticed that if he could construct a compact set $F$ of plane Lebesgue measure zero containing a line segment in every direction this would lead to a counterexample as follows. Assume (by translating $F$ if necessary), that $F$ contains no segment parallel to and of rational distance from either of a fixed pair of axes. Let $f$ be the characteristic function of the set $F_{r}$ consisting of those points of $F$ with at least one rational coordinate. As $F$ contains a segment in every direction on which both $F_{r}$ and its complement are dense, there is a segment in each direction in which $f$ is not Riemann integrable. On the other hand, the set of points of discontinuity of $F$ is of plane measure zero, so $f$ is Riemann integrable over the plane by the well-known criterion of Lebesgue.

The basic idea underlying the original construction of Besicovitch is to form a figure obtained by splitting an equilateral triangle of unit height into many smaller triangles of the same height by dividing up the base, and then sliding these elementary triangles varying distances along the base line. In 1964 Besicovitch developed a completely different approach, using the projection theorem due to Marstrand.

2.1. Some fundamental properties of plane sets of fractional dimension. In this 1954 paper, which was essentially the work for his doctoral thesis at Oxford, and was heavily influenced by Besicovitch, John Marstrand proved the following fundamental result.

\footnotetext{
${ }^{8}$ The virtual collapse of the Russian currency appears to have had nothing to do with it. In 1924, together with Tamarkin, Besicovitch crossed the Soviet border with Norway on foot and made his way to Copenhagen to work with H. Bohr eventually settling in Cambridge in 1927, where, in due course, he became the Rouse Ball Chair. Besicovitch's command of English remained stationary from his early days in Cambridge ('It's a story...'); for him, for example, the definite article was superfluous. A story is told that during one of his lectures an undergraduate tittered at some distortion of English idiom. 'Gentlemen,' said Besicovitch, 'there are 50 million Englishmen speak English you speak; there are 500 million Russians speak English I speak.'
} 
Theorem 1 (Marstrand's Projection Theorem). Denote the projection in the direction $\theta$ by $\pi_{\theta}$. If $X \in \mathbb{R}^{2}$ is a Borel subset of Hausdorff dimension $s$, then $\operatorname{dim}_{H}\left(\pi_{\theta} X\right)=\min (s, 1)$ for almost every $\theta$.

Concerning the finer information about the set of exceptional $\theta$ in Theorem 1, Kaufman proved that if $\operatorname{dim} X \geq t$, $B \subset S^{1}$ with $\operatorname{dim} B>t$, then there exists $\theta \in B$ such that $\operatorname{dim}\left(\pi_{\theta}(X)\right) \geq t$. Using crucially (1), in "The discretized sum-product and projection theorems,' Bourgain established the following, sharper result.

Theorem 2. Given $0<\alpha<2$ and $\kappa>0$, there is $\eta>\frac{\alpha}{2}$ such that, if $X \subset \mathbb{R}^{2}$ is of Hausdorff dimension greater than $\alpha$, then $\operatorname{dim}_{H}\left(\pi_{\theta}(X)\right) \geq \eta$ for all $\theta \in S^{1}$ except in an exceptional set $E$ satisfying $\operatorname{dim}_{H}(E) \leq \kappa$.

\subsection{Besicovitch type maximal operators and applica-} tions to Fourier Analysis.

We must admit with humility that, while number is purely a product of our mind, space has a reality outside of our mind, so that we cannot prescribe its laws a priori.

Gauss, Letter to Bessel, 1830

The Kakeya problem in $\mathbb{R}^{n}$ is to estimate the fractal dimension of the Besicovitch set $E \subset \mathbb{R}^{n}$, i.e., a set containing line segments of length one in all directions.

Conjecture 1. Let $E$ be a Besicovitch set in $\mathbb{R}^{n}$. Then $\beta(n)=$ $\operatorname{dim}(E)=n$.

There are several relevant notions of 'fractal dimension,' the simplest being the Minkowski dimension, defined as follows. Let $A$ be a closed subset of a metric space $X$. Fix some radius $\delta$. Let $\mathcal{N}(A, \delta)$ be the least number of balls of radius $\delta$ needed to cover $A$. If $A$ is a rectifiable curve in $\mathbb{R}^{n}$ it is easy to see that $\mathcal{N}(A, \delta)$ is of order $\delta^{-1}$. If $A$ is a surface, $\mathcal{N}(A, \delta)$ is approximately $\delta^{-2}$. This suggests the idea of defining the dimension of an arbitrary set as the number $d$ for which $\mathcal{N}(A, \delta) \sim \delta^{-d}$. The limit

$$
\lim _{\delta \rightarrow 0} \frac{\log \mathcal{N}(A, \delta)}{\log \left(\delta^{-1}\right)}
$$

if it exists, is called Minkowski dimension, $\operatorname{dim}_{M}(A)$.

The basic result proved by Davies in 1971 is that $\beta(2)=$ 2. The same year $\mathrm{C}$. Fefferman discovered the intimate connection between the Kakeya problem and the multiplier problem for the ball, proving that for $d \geq 2$ the map $f \rightarrow \int_{|\xi| \leq 1} \hat{f}(\xi) e^{i x \xi} d \xi$ defines only for $p=2$ a bounded operator on $L^{p}\left(\mathbb{R}^{d}\right)$. This seminal result made apparent the fundamental connection between Kakeya-type questions and the higher-dimensional Fourier Analysis, in particular in the theory of oscillatory integral operators. ${ }^{9}$

${ }_{9}^{9}$ A recent triumph in this area is the resolution of the Vinogradov's conjecture by Bourgain, Demeter, and Guth, establishing near-optimal bounds on the mean
In the 1980s Drury and Christ showed that

$$
\beta(n) \geq \frac{n+1}{2} \text {. }
$$

The argument consists of intersecting the line segment $L_{\xi} \subset E, L_{\xi}$ parallel to $\xi$ in $S^{d-1}$, by a pair of parallel hyperplanes $H_{1}, H_{2}$ in $\mathbb{R}^{d}$ and observing that for all $\delta>0$

$$
\left(\frac{1}{\delta}\right)^{d-1} \lesssim \mathcal{N}\left(H_{1} \cap E, \delta\right) \mathcal{N}\left(H_{2} \cap E, \delta\right)
$$

The estimate (2) was first improved by Bourgain in 1991, in the paper eponymous with the title of this subsection, to $\frac{n+1}{2}+\varepsilon_{n}$ with $\varepsilon_{n}$ given by a recursive argument (for $n=3$ this yields bound $\frac{7}{3}$ ) by using a 'bush' argument. A more efficient geometric argument, using 'hairbrushes,' was given several years later by $\mathrm{T}$. Wolff, leading to

$$
\operatorname{dim}_{H}(E) \geq \frac{n}{2}+1 \text {. }
$$

The space constraints prevent me from going into the details of these arguments; referring the reader to beautiful surveys by Izabella Łaba, Terence Tao and Thomas Wolff, I will restrict myself to two remarks.

The first remark is that these developments made apparent the connection between Kakeya-type problems and results in combinatorial geometry, such as the SzemerédiTrotter Theorem, which will be briefly discussed in section 3.2.2.

The second remark is that Bourgain's interest in the Kakeya problem was stimulated by his discovery of it being implied by the following version of Montgomery's conjecture $^{10}$ for Dirichlet polynomials.

Conjecture 2. Let $S(s)=\sum_{n=1}^{N} a_{n} n^{s}$ with $\left|a_{n}\right| \leq 1$, and let $\mathcal{F}$ be a set of 1-separated reals in the interval $[0, T], T>N$. Then

$$
\sum_{t \in \mathcal{F}}|S(i t)|^{2} \ll T^{\mathcal{\varepsilon}}(N+|\mathcal{F}|) N\left(\max _{1 \leq n \leq N}\left|a_{n}\right|^{2}\right) .
$$

Regrettably skipping thus over many important and pertinent developments that took place in the last decade of the past century, let us note, looking forward, that in its

values of exponential sums such as

$$
\sum_{n=1}^{N} e^{2 \pi i\left(\alpha_{1} n+\alpha_{2} n^{2}+\cdots+\alpha_{k} n^{k}\right)}
$$

as one varies the frequencies $\alpha_{1}, \ldots, \alpha_{k}$; these are of fundamental importance in analytic number theory.

${ }^{10}$ One of the consequences of Montgomery's conjecture is the density hypothesis for the Riemann zeta function

$$
N(\sigma, T) \ll T^{2(1-\sigma)+\varepsilon}
$$

Here $\frac{1}{2}<\sigma<1, T>0$ and $N(\sigma, T)$ is the number of zeros $\rho=\beta+i \gamma$ of $\zeta(s)$ satisfying $\beta>\sigma,|\gamma|<T$. 
closing year (1999) Bourgain unveiled the connection between the Kakeya problem and one of the most consequential and far-reaching results in arithmetic combinatorics, obtained by Gowers in his groundbreaking 'New proof of Szemerédi's Theorem for arithmetic progressions of length four.' This result, the Balog-Szemerédi-Gowers Lemma, will play a crucial role in many subsequent developments, some of which are discussed in this essay.

\subsection{Balog-Szemerédi-Gowers Lemma.}

Either this universe is a mere confused mass, and an intricate context of things, which shall in time be scattered and dispersed again; or it is a union consisting of order and administered by Providence.

Marcus Aurelius 'Meditations' 6, VIII Complete disorder is impossible.

T.S. Motzkin

The Balog-Szemerédi-Gowers Lemma is ostensibly a statement about group structure, but the main tool in its proof is a remarkable (and remarkably useful) graphtheoretic result best viewed in the context of Ramsey theory. Ramsey theory is a systematic study of the following general phenomenon. Surprisingly often, a large structure of a certain kind has to contain a fairly large highly organized substructure, even if the structure itself is completely arbitrary and apparently chaotic. It can be viewed as a vast generalization of the pigeonhole principle, which states that if a set $X$ of $n$ objects is colored with $S$ colors, then there must be a subset of $X$ of size at least $\frac{n}{s}$ that uses just one color. Such a subset is called monochromatic. The situation becomes more interesting if the set $X$ has some additional structure. It then becomes natural to ask for a monochromatic subset that keeps some of the structure of $X$. However it also becomes much less obvious if such a subset exists. Frank Plumpton Ramsey in 1930 took as his set $X$ the set of all the edges in a complete graph and the monochromatic subset he obtained consisted of all the edges of some complete graph. One version of his theorem is as follows. For every positive integer $k$ there is a positive integer $N$ such that if the edges of the complete graph are all colored either red or blue, then there must be $k$ vertices such that all edges joining them have the same color. That is, a sufficiently large complete graph colored with two colors contains a complete subgraph of size $k$ which is monochromatic. The least integer $N$ that works is known as $R(k)$, and it is known that

$$
2^{\frac{k}{2}} \leq R(k) \leq 2^{2 k} .
$$

There were several results in Ramsey theory predating Ramsey's Theorem, in particular van der Waerden proved that if you color the integers with some finite number $r$ of colors, there must be some color that contains arithmetic progressions of every length. In 1935 Erdös and Turán conjectured that this holds for 'the most popular' color class.
More precisely, they conjectured that for any positive integer $k$ and any real number $\varepsilon>0$ there is a positive integer $n_{0}$ such that if $n>n_{0}$ any set of at least $\varepsilon n$ positive integers between 1 and $n$ contains a $k$-term arithmetic progression. This conjecture was proved by Szemerédi in 1975 using, amongst other things, his celebrated Regularity Lemma, which can be very roughly described as a statement that even the most 'chaotic' systems can be decomposed into a 'relatively' small number of 'approximately regular' subsystems.

Using the Szemerédi Regularity Lemma the following result was established by Balog and Szemerédi in 1994, resulting in tower-like exponential type dependence (cf. (7)). Gowers achievement of the polynomial bounds $K^{O(1)}$ in the statement below is crucial in the ensuing applications.

Theorem 3 (Balog-Szemerédi-Gowers Lemma). Let $\mathcal{G}(A, B, E)$ be a finite bipartite graph, that is, a graph whose vertices can be partitioned into two disjoint sets, with $|E| \geq$ $\frac{|A||B|}{K}$. Then there exist subsets $A^{\prime} \subset A$ and $B^{\prime} \subset B$ with $\left|A^{\prime}\right| \gg K^{-O(1)}|A|$ and $\left|B^{\prime}\right| \gg K^{-O(1)}|B|$ such that for every $a \in A$ and $b \in B$, $a$ and $b$ are joined $b y \gg K^{-O(1)}|A| B \mid$ paths of length three.

The fact that the following corollary is valid for noncommutative groups was established by Tao.

Corollary 4. Let $A, B$ be finite nonempty subsets of a group $G$ and suppose

$$
\left\|1_{A} \star 1_{B}\right\|_{l^{2}(G)} \geq \frac{|A|^{\frac{3}{4}}|B|^{\frac{3}{4}}}{K}
$$

for some $e^{11} K \geq 1$. Then there exist subsets $A^{\prime} \subset A$ and $B^{\prime} \subset B$ with $\left|A^{\prime}\right| \gg K^{-O(1)}|A|$ and $\left|B^{\prime}\right| \gg K^{-O(1)}|B|$ with $\left|A^{\prime} \cdot B^{\prime}\right| \ll$ $K^{O(1)}|A||B|$ and $\left|A^{\prime} \cdot\left(A^{\prime}\right)^{1}\right| \ll K^{O(1)}|A|$.

The quantity $\left\|1_{A} \star 1_{B}\right\|_{l^{2}(G)}$ counts the number of solutions to the equation $a_{1} \cdot b_{1}=a_{2} \cdot b_{2}$ with $a_{1}, a_{2} \in A$ and $b_{1}, b_{2} \in B$ (multiplicative or additive quadruples) and is also known as the multiplicative energy of $A$ and $B$.

2.4. On the dimension of Kakeya sets and related maximal inequalities. The main result in this 1999 paper of Bourgain is the following improvement of (4) for large $n$ :

$$
\operatorname{dim}_{H}(E) \geq \frac{1}{25}(13 n+12) .
$$

The heart of the argument consists in applying the Balog-Szemerédi-Gowers Lemma to show that Kakeya set $E$ satisfies $\mathcal{N}_{\delta} \geq \delta^{-\alpha(n-1)}$ with $\alpha>\frac{1}{2}$ as follows. Let $L$ be the lattice $\delta \mathbb{Z}^{n} \subset \mathbb{R}^{n}$, and for each of the segments $\left\{x+t e:|t| \leq \frac{1}{2}\right\}$ with $e \in S^{n-1}$ in the definition of Kakeya

\footnotetext{
${ }^{11}$ Here $\star$ denotes the convolution operation: $f \star g=\int_{G} f(y) g\left(y^{-1} x\right) d \mu(y)$. Note that by Young's inequality $\left\|1_{A} \star 1_{B}\right\|_{l^{2}(G)} \leq|A|^{\frac{3}{4}}|B|^{\frac{3}{4}}$.
} 


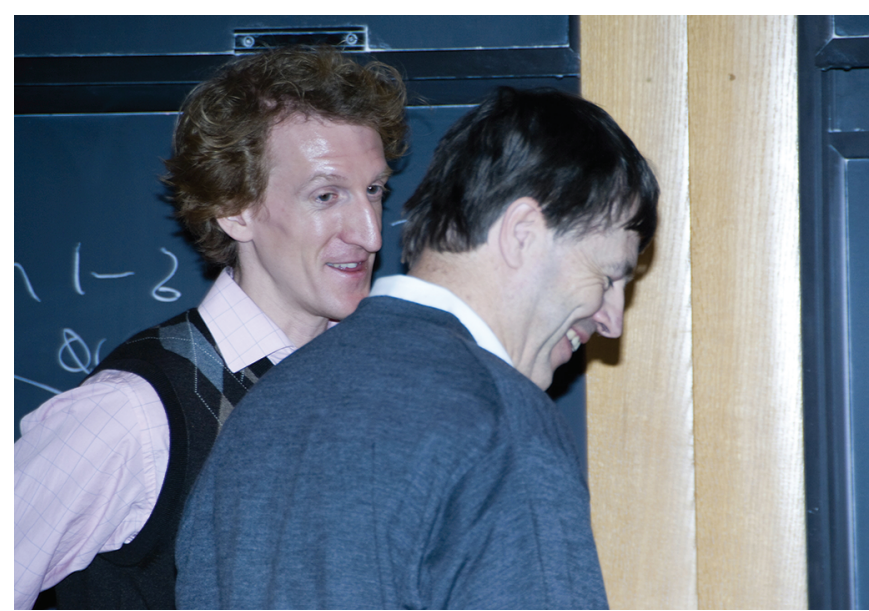

Figure 3. Jean Bourgain and Ben Green.

set, let $x^{+}$and $x^{-}$be the elements of $L$ closest to $x+\frac{1}{2} e$ and $x-\frac{1}{2} e$, respectively. Let $A$ be the set whose elements are the various $x^{+}$and $x^{-}$and define $\mathcal{G} \subset A \times A$ to be the set of pairs $\left(x^{+}, x^{-}\right)$; then let $S$ be the set of sums $x^{+}+x^{-}$. Clearly $|A| \lesssim \mathcal{N}_{\delta}(E)$, and in addition $|S| \lesssim \mathcal{N}_{\delta}(E)$, since the midpoint $\frac{1}{2}\left(x^{+}+x^{-}\right)$is within $C \delta$ of $x \in E$. But it is equally clear that a point of $\mathbb{P}^{n-1}$ is within $C \delta$ of some difference $x^{+}-x^{-}$. Thus $\delta^{-(n-1)} \lesssim \mathcal{N}_{\delta}(E)^{2-\varepsilon}$, as claimed.

This paper marked the first application of Additive Combinatorics to Harmonic Analysis. ${ }^{12}$

\section{Sum-Product Phenomena and the Labyrinth of the Continuum}

Additive Combinatorics grew out of the classical additive number theory. Though few isolated results existed before, the turning point was Shnirelmann's approach to Goldbach's conjecture asserting that any integer greater than three can be expressed as a sum of two or three primes, depending on parity. Shnirelmann proved the weaker result that there is a bound $k$ so that every integer is a sum of at most $k$ primes, or, in other words, the primes form an additive basis. Shnirelmann's approach, notwithstanding it being soon superseded for the Goldbach's problem by Vinogradov's method of exponential sums, kindled the interest in addition of general sets; a result of fundamental and lasting importance in this subject is due to G. Freiman,

\footnotetext{
12'Bourgain's argument was, to this author's knowledge, the first application of additive number theory to Euclidean harmonic analysis. It was significant, not only because it improved Kakeya bounds, but perhaps even more so because it introduced many harmonic analysts to additive number theory, including Tao, who contributed so much to the subject later on, and jump-started interaction and communication between the two communities. The Green-Tao Theorem and many other developments might never have happened were it not for Bourgain's brilliant leap of thought in 1998.' Izabella Łaba, BAMS, 2008.
}

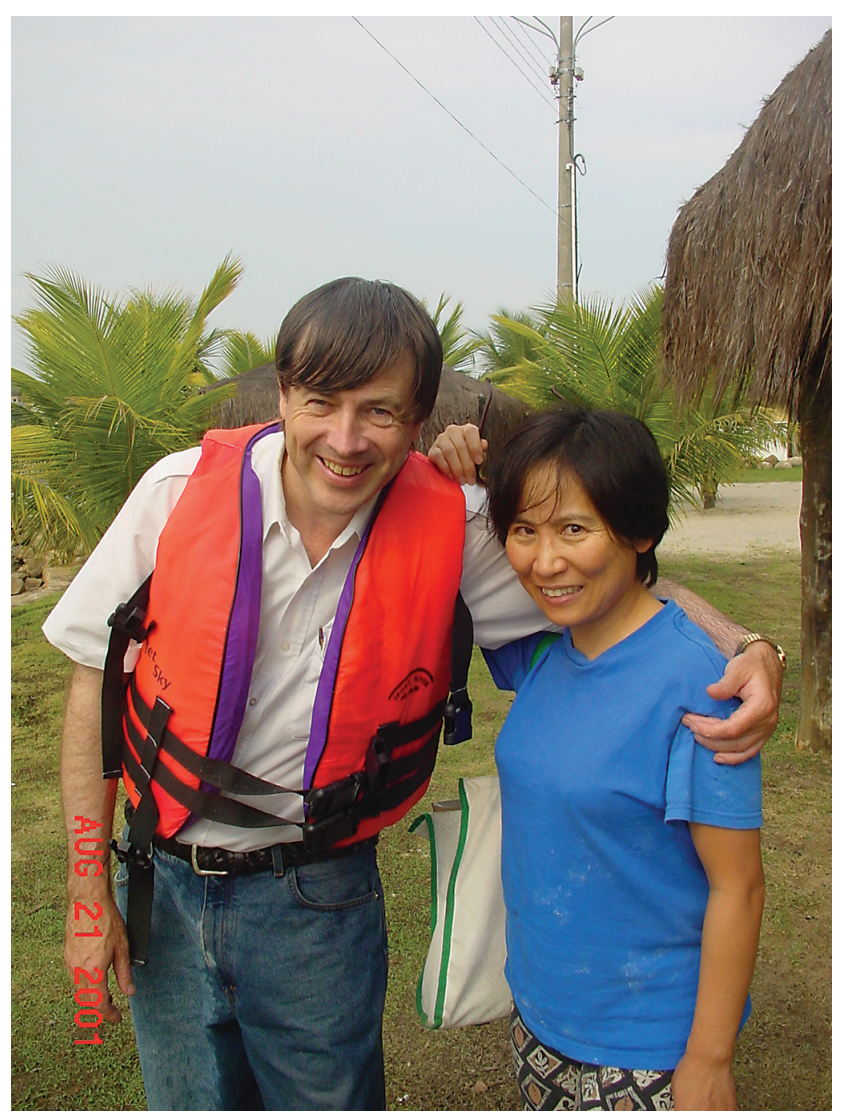

Figure 4. Jean Bourgain and Mei-Chu Chang.

a student of Gelfond, who was a close friend and collaborator of Shnirelmann. ${ }^{13}$

3.1. Freiman's Theorem and Ruzsa's calculus. Freiman's Theorem gives a characterization of sets with small doubling in terms of generalized arithmetic progressions. A $d$-dimensional generalized arithmetic progression (GAP) is a set $P$ of the form

$$
\left\{a+x_{1} q_{q}+\cdots+x_{d} q_{d}: 0 \leq x_{i} \leq l_{i}\right\},
$$

where $l_{1}, \ldots, l_{d}$ are positive integers. We call $d$ the dimension of $P$; by the size of $P$ we mean $\|P\|=\prod_{i=1}^{d}\left(l_{i}+1\right)$, which is the same as the number of elements if all sums in (10) are distinct (in which case we say that $P$ is proper). Note that

$$
|P+P|<2^{d}|P| \leq 2^{d}\|P\|
$$

Theorem 5 (Freiman's Theorem). If $A \subset \mathbb{Z},|A|=n$, $|A+A| \leq \alpha n$, then $A$ is contained in a generalized arithmetic progression of dimension at most $d(\alpha)$ and size at most $s(\alpha) n$.

\footnotetext{
${ }^{13}$ Shnirelmann committed suicide on 24 September 1938, fearing imminent persecution by NKVD (subsequently KGB; currently FSB).
} 
The quantitative bound in Freiman's Theorem, used by Bourgain in his first proof of (1), is due to Mei-Chu Chang: ${ }^{14} d<\alpha$ (the best possible) and $s \leq e^{\alpha^{c}}$.

Freiman's proof was considerably simplified by Ruzsa (building on the earlier work of Plünnecke). One of the fundamental notions introduced by Ruzsa is that of Ruzsa distance between two sets $X$ and $Y$ in a group, $\rho(X, Y)=$ $\log \frac{|X-Y|}{\sqrt{|X||Y|}}$, allowing us to rewrite an elementary inequality for $A, Y, Z$ finite sets in a group (which, as observed by Tao, is not necessarily commutative) $|A||Y-Z| \leq|A-Y||A-Z|$ as

$$
\rho(Y, Z) \leq \rho(Y, A)+\rho(A, Z),
$$

a triangle inequality-like property; $\rho$ is also symmetric (but $\rho(X, X)$ is typically positive). The following result of Plünnecke and Ruzsa was used in Bourgain's $2+$ proof in place of Freiman's Theorem.

Theorem 6. Let $A, B$ be finite sets in a group and write $|A|=$ $m,|A+B|=\alpha m$. For arbitrary nonnegative integers $k, l$ we have

$$
|k B-l B| \leq \alpha^{k+l} m
$$

3.2. Sum-product phenomena and incidence geometry. Freiman's Theorem is an example of an 'inverse' result: knowing that the set has small doubling we can characterize its structure in terms of GAPs. One of the basic 'direct' results, applicable to arbitrary sets, is the 'sumproduct phenomenon,' whose elementary and elemental nature might be described as follows. When studying addition and multiplication tables for numbers from 1 to 9 one might notice that there are many more numbers in the multiplication table. This basically has to do with the fact that the numbers from one to nine form an arithmetic progression. If you take a set forming an arithmetic progression (or a subset of it) and add it to itself it will not grow much; if you take a set forming a geometric progression (or a subset of it) and multiply it by itself it will also not grow much. However a subset of integers cannot be both an arithmetic and a geometric progression and so it will grow either when multiplied or added with itself.

In 1983 Erdös and Szemerédi proved that for any finite set of integers $A$

$$
|A+A|+|A \cdot A| \geq C|A|^{1+\varepsilon}
$$

for absolute constants $C, \varepsilon$ and conjectured that in fact for any $\varepsilon>0$ there is $C_{\varepsilon}$ such

$$
|A+A|+|A \cdot A| \geq C_{\varepsilon}|A|^{2-\varepsilon} .
$$

We will give a beautiful proof (due to Elekes and Székeley) of (14) with $\varepsilon=\frac{3}{4}$ using the Szemerédi-Trotter Theorem in incidence geometry, mentioned in section 2.2,

\footnotetext{
${ }^{14}$ It is known that a bound for $s$ must be $\gg 2^{\alpha}$; very likely the proper order is $e^{c \alpha}$. A beautiful survey by T. Sanders in BAMS covers the recent developments.
}

which in turn will follow from the crossing number inequality obtained, ultimately, from a purely topological result: Euler's formula.

3.2.1 Crossing number inequality. During World War II, Turán worked as forced labor, moving wagons filled with bricks from kilns to storage places. According to his recollections, it was not a very tough job, except that they had to push much harder at the crossings. This led him to consider the following problem: for a nonplanar graph $\mathcal{G}$, find a drawing for which the number of crossings is minimal. The minimal number of crossings in a drawing is called the crossing number of a graph $\operatorname{Cr}(\mathcal{G})$. Another practical application of this problem appeared in the early eighties, when it turned out that the chip area required for the realization of an electrical circuit (VLSI layout) is closely related to the crossing number of the underlying graph. The basic result, due to Leighton, is as follows:

$$
\operatorname{Cr}(\mathcal{G}) \geq \frac{1}{64} \frac{|E|^{3}}{|V|^{2}}-|V|
$$

Here $|V|$ and $E$ denote, respectively, the number of vertices and edges in the graph. The proof starts by observing that Euler's formula implies that if $\operatorname{Cr}(\mathcal{G})=0$, then $|V|-|E|+$ $|F|=2$. This readily implies that the crossing number of any graph satisfies

$$
\operatorname{Cr}(\mathcal{G}) \geq|E|-3|V|+6 .
$$

The proof is concluded by considering a planar embedding of $\mathcal{G}$ with least crossing number and choosing each vertex of $\mathcal{G}$ at random with probability $p$. Taking the expectations of the relevant quantities gives

$$
p^{4} \operatorname{Cr}(\mathcal{G}) \geq p^{2}|E|-3 p|V|+6 ;
$$

letting $p=\frac{4|E|}{|V|}$ yields the desired inequality (15).

3.2.2 Szemerédi-Trotter Theorem. This is an assertion that given $n$ points and $m$ lines in the plane the number of incidences is

$$
I(m, n) \ll m^{\frac{2}{3}} n^{\frac{2}{3}}+m+n
$$

(and this is sharp). Consider a set $P$ of $m$ points and a set $L$ of $n$ lines in the plane, realizing the maximal number of incidences $I(m, n)$. Define a drawing of a graph $\mathcal{G}(V, E)$ in the plane: each point $p \in P$ becomes a vertex of $\mathcal{G}$ and two points $p, q \in P$ are connected by an edge if they lie on a common line $l \in L$ next to one another. If a line $l \in L$ contains $k \geq 1$ points of $P$, then it contributes $k-1$ edges to $P$ and hence $I(m, n)=|E|+n$. Since the edges are parts of the lines, at most $\left(\begin{array}{l}n \\ 2\end{array}\right)$ pairs may cross: $\operatorname{Cr}(\mathcal{G}) \leq\left(\begin{array}{l}n \\ 2\end{array}\right)$. By the crossing number theorem, $\operatorname{Cr}(\mathcal{G}) \geq \frac{1}{64} \frac{|E|^{3} \mid}{m^{2}}-n$, so $\frac{1}{64} \frac{|E|^{3} \mid}{m_{2}^{2}}-n \leq \operatorname{Cr}(\mathcal{G}) \leq\left(\begin{array}{l}n \\ 2\end{array}\right)$ and a calculation gives $|E|=$ $O\left(m^{\frac{2}{3}} n^{\frac{2}{3}}+m\right)$, proving (16). 
3.2.3 Proof of the sum-product inequality. We are ready to prove (13) with $\varepsilon=\frac{1}{4}$. Let $P=\{(a, b) \mid a \in$ $A+A, b \in A \cdot A\} ; P$ is a subset of the plane and has cardinality $|A+A||A \cdot A|$. Consider the set of lines of the form $\{(x, y): y=a(x-b)\}$, where $a, b$ are elements of $A$. Clearly $L$ has $|A|^{2}$ elements. Moreover, each such line contains at least $|A|$ points in $P$, namely the points $(b+c, a c)$ with $c \in P$. Thus $I(P, L) \geq|A|^{3}$. Applying the Szemerédi-Trotter Theorem and elementary linear algebra we conclude

$$
|A+A|+|A \cdot A|=\Omega\left(|A|^{\frac{5}{4}}\right) .
$$

Before turning to the discussion of Erdös-Volkmann and Katz-Tao discretized ring conjectures, let us note that if the set $A$ is $\delta$-separated, by carefully adapting the preceding proofs we obtain an inequality of the form

$$
\mathcal{N}\left(A+A, \delta^{2}\right)+\mathcal{N}\left(A \cdot A, \delta^{2}\right)>\mathcal{N}(A, \delta)^{1+\tau},
$$

to be contrasted with Bourgain's result (1).

3.3. On the Erdös-Volkmann and Katz-Tao discretized ring conjectures.

\subsubsection{Erdös-Volkmann problem.}

With Volkmann we proved that for every $0 \leq \alpha \leq 1$ there is a group of real numbers of $\operatorname{dim}_{H}=g a$. All our efforts so far failed in proving the existence of ring or field of Hausdorff dimension $\alpha$.

$$
\text { P. Erdös, }{ }^{15} 1979
$$

In 1964 Erdös and Volkmann proved that for each $\alpha$ in $(0,1)$ there is an additive Borel subgroup of the reals with Hausdorff dimension $\alpha$. Several proofs of this fact have now been given, all involving some sets of numbers which are well approximated by rationals. It is a well-known result that there exist infinitely many rational approximations $\frac{m}{n}$ to any real number $r$ with an error less than $n^{-2}$. If $\alpha>2$, let $E$ be the set of real numbers $r$ that can be 'well approximated' by rational numbers in the sense that there are infinitely many rational numbers $\frac{m}{n}$ with $\left|r-\frac{m}{n}\right|<\frac{1}{n^{\alpha}}$. Jarnik proved ${ }^{16}$ in 1931 that $\operatorname{dim}_{H}(E)=\frac{2}{\alpha}$. Falconer's construction ${ }^{17}$ of an additive Borel subset with Hausdorff dimension $\alpha$ builds on Jarník's Theorem: take $n_{k}$ a sequence of positive integers which increases sufficiently rapidly, for

\footnotetext{
${ }^{15}$ Erdös expressed a similar sentiment in a letter to K. Falconer (reproduced with his kind permission). We remark that in 2016 P. Mauldin showed that assuming the Continuum Hypothesis, there exist subrings (and even subfields) of $\mathbb{R}$ of arbitrary Hausdorff dimension, which are not however Borel subsets.

${ }^{16}$ In fact, Jarnik also proved a two-dimensional version of this theorem, yielding a set in $\mathbb{R}^{2}$ which, as was shown by Kaufmann, has the maximal possible set of exceptional projections discussed in section 2.1.

${ }^{17}$ Erdös and Volkmann based their construction of $G_{\alpha}$ on the following beautiful characterization of (ir)rationals given by Cantor (1869): let $x=[x]+$ $\sum_{k=2}^{\infty} \frac{a_{k}(x)}{k !}$ with the integers $a_{k}(x)$ satisfying $0 \leq a_{k}(x) \leq k-1$. Then $x$ is irrational iff $a_{k}(x)>0$ for infinitely many $k$ and $a_{k}(x) \leq k-1$ for infinitely many $k$. For fixed $\alpha$ their $G_{\alpha}$ consists of those $x$ which satisfy $a_{k}(x) \leq \kappa(x) k^{\alpha}$ or $a_{k}(x) \geq k-\kappa(x) k^{\alpha}$ for all $k \geq k_{0}(x)$ and $\kappa(x)$ positive constant.
}

example $n_{k+1}>n_{k}^{k}$. Define the set $G_{\alpha}$ to consist of those real numbers for which there exists $M$ such that for any $k$ there is an integer $p$ such that $\left|x-\frac{p}{n_{k}}\right|<M n_{k}^{-\frac{1}{\alpha}}$. Clearly $G_{\alpha}$ is an additive subgroup and it is not difficult to show, using Jarnik's Theorem that its Hausdorff dimension is equal to $\alpha$.

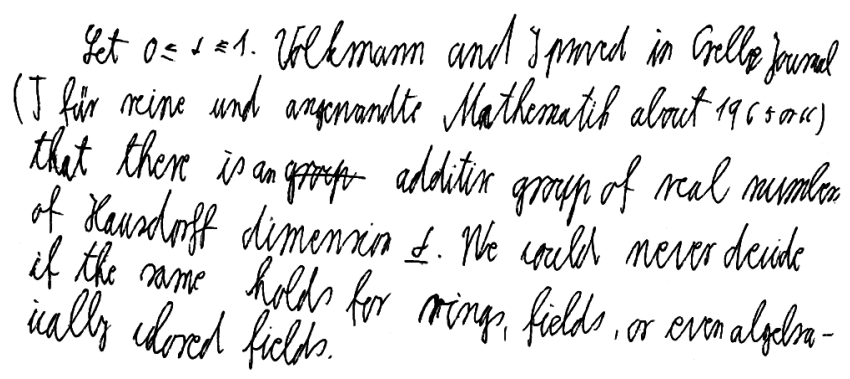

Figure 5. From a letter from P. Erdös to K. Falconer dated 18 June 1983.

3.3.2 Katz-Tao discretized ring conjecture. It was shown by Falconer that a Borel subring $R$ of $\mathbb{R}$ cannot have Hausdorff dimension exceeding $\frac{1}{2}$ (by considerations of the distance set $\{|a-b| ; a, b, \in R \times R\} \subset \sqrt{R})$.

In the 2001 paper 'Some connections between Falconer's distance set conjecture, and sets of Furstenbqerg type,' motivated, in part, by connections with the Kakeya problem, Nets Katz and Terence Tao formulated a quantitative version of the Erdös-Volkmann problem (discretized ring conjecture). A bounded subset $A$ of $\mathbb{R}$ is called a $(\delta, \sigma)_{1}$ set provided $A$ is a union of $\delta$-intervals and satisfies

$$
|A \cap I|<\left(\frac{r}{\delta}\right)^{1-\sigma} \delta^{1-\varepsilon}
$$

whenever $I \subset \mathbb{R}$ is an arbitrary interval of size $\delta \leq r \leq 1$ $(0<\varepsilon \ll 1$ in (19) is a small parameter).

Katz and Tao conjectured that if $A$ is a $\left(\delta, \frac{1}{2}\right)_{1}$ set satisfying $|A|>\delta^{\frac{1}{2}+\varepsilon}$, then necessarily $|A+A|+|A \cdot A|>\delta^{\frac{1}{2}-c}$, with $c>0$ an absolute constant. This was proved by Bourgain in the paper eponymous with the title of this section. More generally, he proved the following result (which is the precise formulation of (1)).

Theorem 7. If $A$ is $a(\delta, \sigma)_{1}$ set, $0<\sigma<1$, satisfying $|A|>$ $\delta^{\sigma+\varepsilon}$, then necessarily $|A+A|+|A \cdot A|>\delta^{\sigma-c}$, with an absolute constant $c=c(\sigma)>0$.

3.3.3 Labyrinth of the continuum. The title of this subsection is described by Bourgain in the introduction to his paper in the bold sentence below.

The statement in Theorem 7 is thus a purely combinatorial fact. We proceed by contradiction, assuming

$$
|A+A|+|A \cdot A|<\delta^{\sigma-c} .
$$




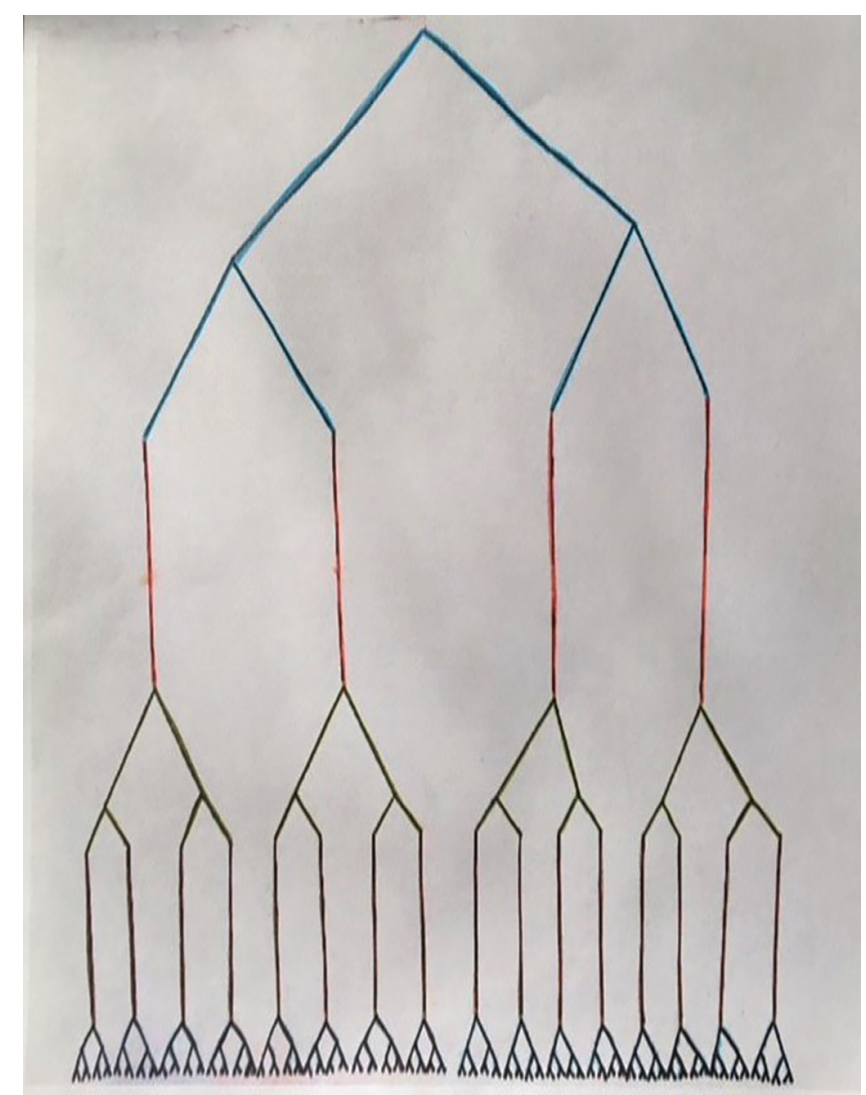

Figure 6. Labyrinth of the continuum.

The initial stages of the argument use only the additive information, thus $|A+A|<\delta^{\sigma-c}$. It is processed through multiscale construction, based on Ruzsa's sumset estimates, and, most importantly, quantitative versions of Freiman's famous theorem on finite sets of reals with small doubling set. ... The final product is a subset $C$ of $A$ with a tree structure which exhibits a 'multiscale porosity property.' At this point, we start using multiplicative structure and prove the existence of elements $x_{1}, x_{2} \in A-A$ such that $\left|x_{1} C+x_{2} C\right|>\delta^{\sigma-\kappa}$.

The key difficulty comes from the fact that Freiman's Theorem describes the structure of sets of small doubling $|A+A|<C|A|$ with a fixed constant $C$, whereas the assumption (20) deals with the situation where the constant $C$ grows with $A$, as $A$ itself increases in size: the heart of Bourgain's argument is the structure theorem characterizing sets satisfying (20). The additive subgroups $G_{\alpha}$ described in section 3.3.1 satisfy this assumption; let us look at their structure more closely, concentrating for concreteness on the case $\alpha=\frac{1}{2}$, and giving an alternative description of it as a subset of the binary tree representing the continuum.
Let $P_{n}=\{0, \ldots, n-1\}$, and let

$$
A_{n}=\sum_{i=1}^{n} \frac{1}{2^{i^{2}}} P_{2^{i}}=\left\{\sum_{i=1}^{n} a_{i} 2^{-i^{2}}: 1 \leq a_{i} \leq 2^{i}\right\} .
$$

It is easy to see that the distance between distinct points $x, x^{\prime} \subset A_{n}$ is at least $\frac{1}{4^{n^{2}}}$, such that $x$ has a unique representation as a sum $\sum_{i=1}^{n} a_{i} 4^{-i^{2}}$ with $1 \leq a_{i} \leq 2^{i}$. Each term of the sum $\sum_{i=1}^{n} a_{i} 2^{-i^{2}}$ determines a distinct block of binary digits; it is seen to be GAP (defined in section 3.1) as the image of $P_{2} \times P_{4} \times \cdots \times P_{2^{n}} \rightarrow A_{n}$ given by $\left(x_{1}, \ldots, x_{n}\right) \rightarrow \sum_{i=1}^{n} x_{i} 2^{-i^{2}}$. The rank of this GAP is $n$ so $\left|A_{n}+A_{n}\right| \leq 2^{n}\left|A_{n}\right|$ and $\left|A_{n}\right|=\prod_{i=1}^{n}\left|P_{2^{i}}\right|=2^{\frac{n(n+1)}{2}}$. So we have $\left|A_{n}+A_{n}\right|=\left|A_{n}\right|^{1+o(1)}$.

Now we pass to the limit, akin to the way used in constructing the Cantor set: at stage $n$ we have a collection of $2^{\frac{n(n+1)}{2}}$ intervals of length $2^{-n^{2}}$; from each of these intervals we keep $2^{n+1}$ subintervals of length $2^{-(n+1)^{2}}$ separated by gaps of length $2^{-n^{2}-(n+1)}$. It is easy to see that the resulting fractal set coincides with $G_{\frac{1}{2}}$.

A full binary tree of height $h$ can be identified with a set of 0,1 valued sequences of length $\leq h$. Let us say that the tree $T$ has full branching for $m$ generations at the vertex $\sigma$ if $\sigma$ has all $2^{m}$ possible descendants $m$ generations below it, that is, $\sigma \eta \in T$ for all $\eta \in\{0,1\}^{m}$. The tree is fully concentrated for $m$ generations at $\sigma$ if $\sigma$ has a single descendant $m$ generations down, that is, there is a unique $\eta \in\{0,1\}^{m}$ with $\sigma \eta \in T$. The sets $A_{n}$ are represented by trees $T_{n}$ of height $n^{2}$. For every $i<n$ every node at level $i^{2}$ has full branching for $i$ generations and every node at level $i^{2}+i$ is fully concentrated for $i+1$ generations. Consequently for every $j \in\left[i^{2}, i^{2}+1\right)$ every node at level $j$ has full branching for one generation; for $j \in\left[i^{2}+i,(i+1)^{2}\right)$ every node at level $j$ is fully concentrated for one generation. Moreover, it is not difficult to see that for every $m$ we can partition the levels $0,1, \ldots, n^{2}$ into three sets $U, V, W$ such that:

a. For every $i \in U$ every level $i$ node has full branching for $m$ generations.

b. For every $j \in V$ every level $j$ node is fully concentrated for $m$ generations.

c. The set $W$ constitutes a negligible fraction of the levels: $\frac{|W|}{n^{2}}=o(1)$ as $n \rightarrow \infty$ (with $m$ fixed).

In the above description $U=\bigcup_{i>m}\left[i^{2}, i^{2}+i-m\right), V=$ $\bigcup_{i>m}\left[i^{2}+1,(i+1)^{2}-m\right)$, and $W$ is the set of remaining levels.

Bourgain's Structure Theorem for sets satisfying (20) can now be informally stated as follows. Suppose $|A+A| \sim$ $|A|^{1+\tau}$. If $b \geq 2$ is a base (say $b=2$ ) we can identify $A$ with a subset of the full $b$-ary tree of height $m$ : the vertices at distance $j$ from the root are the intervals $\left[k b^{m-j},(k+1) b^{m-j}\right)$ which intersect $A$. Given $\varepsilon$ there are $\tau>0$ and $b \geq 2$ 


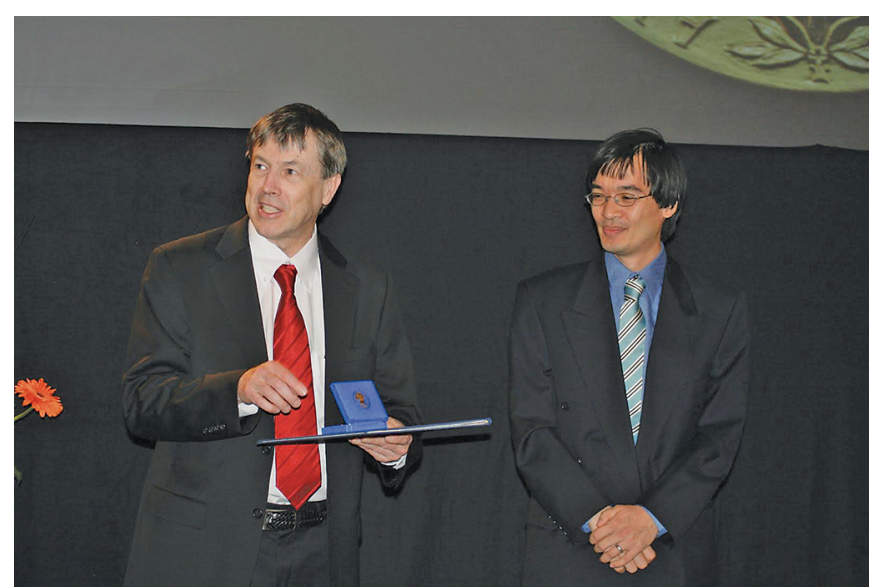

Figure 7. Jean Bourgain and Terence Tao.

(which can be taken arbitrarily large) such that the following holds if $m$ is large enough. Suppose $A \subset\left\{0,1, \ldots, b^{m-1}\right\}$ and $|A+A| \leq b^{\tau m}|A|$ (which is the case if $|A+A| \sim|A|^{1+\tau}$ ). Then there is a subset $A^{\prime}$ of $A$ satisfying the following properties: of $A$.

1. $\left|A^{\prime}\right| \geq b^{\varepsilon m}|A|$, that is to say $A^{\prime}$ is a fairly dense subset

2 . The $b$-ary tree associated with $A^{\prime}$ is regularized in the sense that any vertex at level $j$ has the same number $N_{j}$ of children

3. Either $N_{j}=1$ or $N_{j} \geq b^{1-\varepsilon}$, so at each level the tree has either no branching or close to full branching uniformly over all the vertices at that level.

From Theorem 7 Bourgain deduced that the answer to the Erdös-Volkmann problem was negative, which was proved independently at about the same time by Edgar and Miller who gave a simple and elegant proof making crucial use of Marstrand's Projection Theorem (Theorem 1). The essential idea of their argument served as the starting point and inspiration for the celebrated paper by Bourgain, Katz and Tao establishing the sum-product theorem in $\mathbb{F}_{p}$.

3.4. A sum-product estimate in finite fields and applications. The main result of this paper is the following.

Theorem 8. Let $A$ be a subset of $\mathbb{F}_{p}$ such that for some $\delta>0$

$$
p^{\delta}<|A|<p^{1-\delta} \text {. }
$$

Then

$$
|A+A|+|A \cdot A| \geq c(\delta)|A|^{1+\varepsilon}
$$

for some $\varepsilon=\varepsilon(\delta)>0$.

\section{Here is Terence Tao's recollection:}

Regarding the prehistory of my paper with Jean Bourgain and Nets Katz, it all started with a question of Tom Wolff back in 2000, shortly before his unfortunate death. Tom had formulated the finite field version of the Kakeya conjecture (now solved by Dvir), and had observed that there appeared to be a connection between that conjecture (at least in the 3D case) and what is now the sum-product theorem. (Roughly speaking, if the sum-product phenomenon failed, then one could construct 'Heisenberg group-like' examples that almost behaved like Kakeya sets.) So he posed the question to me (as a private communication) as to whether the sum-product phenomenon was true. Nets and I chewed on this problem for a while, and found connections to some other problems (the Falconer distance problem, and the Szemerédi-Trotter Theorem, over finite fields), but couldn't settle things one way or another. We then turned to Euclidean analogues, and formulated the discretized ring conjecture and showed that this was equivalent to a nontrivial improvement on the Falconer distance conjecture and on a conjecture of Wolff relating to some sets studied by Furstenberg.

After chasing some dead ends on both the finite field sumproduct problem and the discretized ring problem, we gave both problems to Jean, noting that the sum-product problem would likely have applications to various finite field incidence geometry questions, including Kakeya in $\mathbb{F}_{p}^{3}$. Jean managed to solve the discretized ring problem using some multiscale methods, as well as some advanced Freiman Theorem type technology based on earlier work of Jean and Mei-Chu Chang. About the same time, Edgar and Miller solved the qualitative version of the discretised ring problem (i.e. the Erdös ring conjecture).

This left the finite field sum-product problem. All the methods in our collective toolboxes were insensitive to the presence of subfields (except perhaps for Freiman's Theorem, but the bounds were (and still are) too weak to get the polynomial expansion; the multiscale amplification trick that worked in the discretized ring conjecture was unavailable here) and so were insufficient to solve the problem. We knew that it would suffice to show that some polynomial combination of $A$ with itself exhibited expansion, but we were all stuck on how to do this for about a year, until Jean realized that the Edgar-Miller argument (based on the linear algebra dichotomy between having a maximally large span, and having a collision between generators) could be adapted for this purpose. (I still remember vividly the two-page fax from Jean conveying this point. After this breakthrough the paper got finished up quite rapidly. Of course nowadays there are many simple proofs and strengthenings of this theorem, but it was certainly a very psychologically imposing problem for us before we found the solution.

In 2006 Bourgain, Glibichuk and Konyagin proved (22) under the weaker assumption that $|A|<p^{1-\delta}$ and, combining this result with the Balog-SzemerédiGowers Lemma, made remarkable progress towards the Montgomery-Vaughan-Wooley conjecture. This asserts that multiplicative subgroups of $\mathbb{F}_{p}{ }^{*}$ have 'negligible additive structure' as soon as $\frac{|H|}{\log p} \rightarrow \infty$. This was established for $H$ satisfying $|H| \geq p^{\frac{1}{4}+\delta}$ by Konyagin in 2002; Bourgain, Glibichuk and Konyagin proved that the result holds as soon as $|H|>p^{\varepsilon}$ for any $\varepsilon$. Subsequently Bourgain refined and extended this approach to obtain hitherto untouchable estimates for exponential sums pertaining to Diffie-Hellman key exchange, a result of fundamental significance in cryptographic applications. 


\section{Discrete and Continuous Variations on the Expanding Theme}

4.1. Bemerkung über den Inhalt von Punktmengen. Building on Hausdorff's 1914 construction, detailed below, Banach and Tarsky, in 1924, proved that there is a way of decomposing a three-dimensional ball ('precisely measured sphere') into a finite number of disjoint pieces, then reassembling the pieces to form two balls of the same radius, where 'reassembling' means that the pieces are translated and rotated and that they end up still disjoint.

The construction, perhaps one of the most strikingly paradoxical in Mathematics, has its origins in the question posed by Lebesgue in 1904, in the first textbook on integration bearing his name. One of the properties of his integral is the Monotone Convergence Theorem (MCT); is this property really fundamental or does it follow from more familiar integral axioms? Now MCT is essentially equivalent to countable additivity so the question is concerned with the existence of a positive, finitely (but not countably) additive measure on the reals assigning measure one to the unit interval.

In more detail, the problem is to assign a nonnegative real number $f(A)$ to each bounded subset $A \in \mathbb{R}^{n}$ in such a way that

1. $f(E)=1$ if $E$ is the closed unit cube in $\mathbb{R}^{n}$,

2. $f(A)=f(B)$ if $A$ and $B$ are congruent,

3. $f(A \cup B)=f(A)+f(B)$ if $A$ and $B$ are disjoint,

4. $f\left(A_{1} \cup A_{2} \cup \cdots\right)=f\left(A_{1}\right)+f\left(A_{2}\right)+\cdots$ if $A_{1}, A_{2}, \ldots$ is any denumerable sequence of mutually disjoint sets whose union is bounded.

The congruence condition in 2 is as follows: $A$ and $B$ are congruent if there exists an element $g$ in the Euclidean group of distance-preserving transformations in $\mathbb{R}^{n}$ such that $g(A)=g(B)$. The problem of existence of such an $f$ is the $\sigma$-additive measure problem; the problem of existence of $f$ verifying only the first three properties is the finitely additive measure problem.

Lebesgue had left the countably additive measure problem in $\mathbb{R}^{n}$ unresolved; his construction had proved the existence of $f(A)$ for Lebesgue-measurable bounded subsets and had left the existence of nonmeasurable subsets as an open question. This was settled by Vitali in 1905, whose construction is a fore-runner of the Hausdorff-BanachTarsky paradox. Let $l_{\theta}$ by a line segment in $\mathbb{R}^{2}$ given by $l_{\theta}=\{(r, \theta): 0 \leq r \leq 1\}$ in polar coordinates. Consider $\bigcup_{\theta} l_{\theta}=D^{\prime}$, a unit disc with the origin removed. The line segments $l_{\theta}$ and $l_{\phi}$ belong to the same equivalence class if $\theta-\phi$ is a rational multiple of $\pi$. Consider a set $E$ that is a union of a set of $l_{\theta}$ containing exactly one representative from each equivalence class. Rationals are countable: $Q \cap[0,1]=x_{1}, x_{2}, \ldots$. Write $E_{n}=\left\{l_{\theta+2 \pi x_{n}}: l_{\theta} \in E\right\}$. Then each $E_{n}$ is obtained from $E$ by rotation around the origin

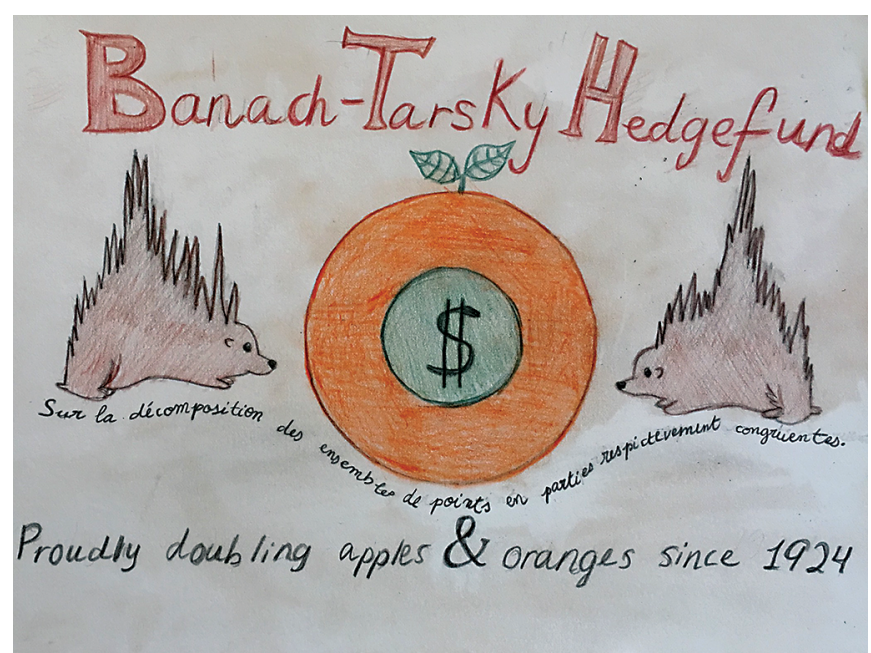

Figure 8.

(by angle $2 \pi x_{n}$ ), the sets $E_{n}$ are disjoint (since $E$ contains representatives from each equivalence class), $\bigcup_{n} E_{n}=D^{\prime}$. Now take $D^{\prime}$ and split it into the set $F$ consisiting of the union of the sets $E_{2 n}$ and the set $G$ consisting of the sets $E_{2 n+1}$. Each $E_{2 n}$ can be rotated to $E_{n}$, and the union of the $E_{n}$ gives us $D^{\prime}$. Similarly, each $E_{2 n+1}$ can be rotated to $E_{n}$, and the union of the $E_{n}$ gives us $D^{\prime}$ again. Thus the punctured unit disc can be split into a countable set of disjoint pieces (all obtained by rotation of one particular set) and translated to form disjoint sets whose union is two copies of $D^{\prime}{ }^{18}$

Hausdorff begins his 1914 paper 'Bemerkung über den Inhalt von Punktmengen' by using the subgroup $G_{\delta}=$ $\{n \delta, n \in \mathbb{Z}\}$ (where $\delta$ is a fixed irrational number) to show that the $\sigma$-additive problem in $\mathbb{R}^{n}$ has no solution for any $n \geq 1$. Both Vitali and Hausdorff use a denumerably dense subgroup of the additive group (in Hausdorff's case the dense group is $G=G_{\delta}+\mathbb{Z}$ ).

He then proceeds to show that the finitely additive measure problem in $\mathbb{R}^{n}$ has no solution if $n \geq 3$ by reducing the problem to the unit sphere $K=S^{2}$ in $\mathbb{R}^{3}$ and then producing the so-called Hausdorff paradoxical decomposition

$$
K=A \cup B \cup C \cup Q,
$$

where $A, B, C, Q$ are four disjoint subsets of $K, Q$ being denumerable and $A \sim B \sim C \sim B \cup C$, the congruence here being under the group of rotations $\mathrm{SO}(3)$.

A decomposition (23) excludes the possibility of having an $\mathrm{SO}(3)$ invariant finitely additive positive measure set function defined for all subsets of $K$ with $f(K)>0$ : indeed for such an $f, f(Q)$ must be zero and $f(A)=f(B)=$ $f(C)=f(B \cup C)=f(B)+f(C)$, whence all of these

\footnotetext{
${ }^{18}$ Vitali's construction makes use of the Axiom of Choice (because we chose one representative from each equivalence class) and the same is true of the BanachTarski construction.
} 
numbers are zero, which is impossible since $0<f(K)=$ $f(A)+f(B)+f(C)$.

The decomposition (23) is obtained by the consideration of a denumerable subgroup $G=G(\theta, \phi)$ of $\operatorname{SO}(3)$ generated by two rotations $\theta, \phi$ such that $\theta^{2}=1, \phi^{3}=1,1$ being the identity map, and such that $\theta, \phi$ satisfy no other nontrivial relations. As observed by von Neumann ${ }^{19}$ the group $G(\theta, \phi)$ is isomorphic to the free product of $\mathbb{Z}_{2}$ and $\mathbb{Z}_{3}$ and must necessarily contain $F_{2}$, the free group on two generators.

This left open the finitely additive problem in $\mathbb{R}^{1}$ and $\mathbb{R}^{2}$; Banach begins his 1923 paper $^{20}$ (giving the title to the next subsection) by showing that in these spaces the finitely additive measure problem does have infinitely many solutions.

\subsection{Sur le problème de la mesure.}

Banach was not a mathematician of finesse, he was a mathematician of power. Inside he combined a spark of genius with that amazing inner imperative, which incessantly whispered to him, as in Verlaine's verse, 'Il n'y a que la glorie ardente du mètier' [There is only one thing: that intense glory of the craft] - and mathematicians know well that their craft depends on the same mystery as the craft of poets.

Hugo Steinhaus ${ }^{21}$

In this seminal paper Banach considers three questions pertaining to the invariance of finitely additive measures. First, he constructs a finitely additive, positive, translationinvariant measure $\mu$ on the family of bounded subsets of $\mathbb{R}$ such that

1. $\mu(A)<\infty$ for every bounded subset of $\mathbb{R}$ (so that $\mu$ gives rise in an obvious way to an element $\mu_{A}$ of $\left.l^{\infty}(A)\right)$,

2. $\mu_{[a, b]}(f)=\int_{a}^{b} f(x) d x$ for every Riemann integrable function $f$ on an interval $[a, b]$,

3. there exists a Lebesgue integrable function $g$ on an interval $[c, d]$ s.t. $\mu_{[c, d]}(g) \neq \int_{c}^{d} g(x) d x$.

\footnotetext{
${ }^{19}$ In his seminal paper 'Zur allgemeinen Theorie des Masses,' which introduced the notion of amenability.

${ }^{20}$ The first equality in this paper appears just below its title: Stefan Banach (Léopol = Lwów).

'If I cared to define the single most prominent characteristic feature of Lvov school, I would mention its interest in the foundation of various theories. What I mean by this is that if one imagines mathematics as a tree, then the Lvov group was devoted to studying roots and trunks, perhaps even the main boughs, with less interest in the side branches, leaves and flowers.' S. Ulam (a student of Banach and co-holder of the patent for the hydrogen bomb.)

${ }^{21}$ H. Steinhaus 'discovered' Banach on the park bench of Cracow Planty promenade, discussing Lebesgue Measure with Otto Marcin Nikodym. (He viewed this as his 'greatest discovery.') Lebesgue visited Lvov in 1938 to receive an honorary doctorate from Jan Kazimierz University (where Steinhaus was at that time Dean of the Faculty). Upon being given a menu in Polish at the celebratory dinner in the famous Scottish Café, Lebesgue looked at the menu for about 30 seconds with utmost seriousness and said, 'Merci, je ne mange que des choses bien définies.' [Thank you, I eat only well-defined things.]
}

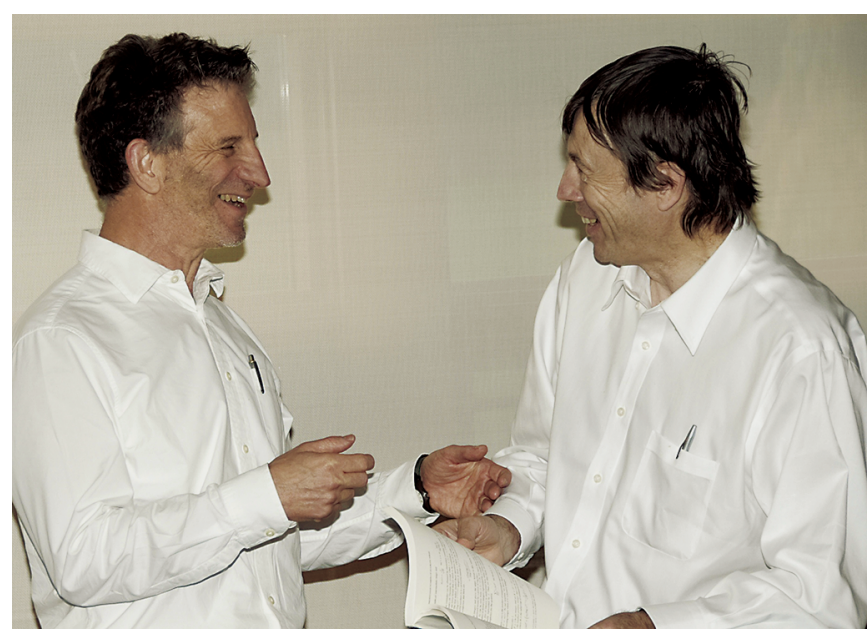

Figure 9. Jean Bourgain and Peter Sarnak.

The second result, which Banach calls 'le probleme large de la mesure,' is to show that unlike the case of $n \geq 3$, studied by Hausdorff, the finitely additive measure problem in $\mathbb{R}^{n}$ for $n=1,2$ does have infinitely many solutions.

The third question, posed by Ruziewicz in 1921, is whether Lebesgue measure on the $n$-sphere is the unique finitely additive rotation invariant measure defined on Lebesgue subsets. Using the Hahn-Banach Theorem, Banach showed that for $n=1$ the answer is negative, using essentially the commutativity of $\mathrm{SO}(2)$. He left the case of $n>2$ open.

For $n>3$ the affirmative answer was obtained in $1980 / 81$ by Margulis and Sullivan who used Kazhdan's property $\mathrm{T}$.

In 1984 Drinfeld established the affirmative answer in the most difficult case of $n=2$ by proving existence of an element in the group ring of $\mathrm{SU}(2)$ which has a spectral gap. As proved by Sarnak, the affirmative answer for $n=2$ implies, via inductive construction, an affirmative answer for $n \geq 2$.

Drinfeld's method used some sophisticated machinery from the theory of automorphic representations, in particular Deligne's solution of Ramanujan's conjecture. In 1986 the explicit and optimal construction, appealing to the above-mentioned tools was obtained by Lubotzky, Phillips and Sarnak, in tandem with their celebrated construction (independently given by Margulis) of Ramanujan graphs.

4.3. Ramanujan-Selberg conjecture. In 1916 Ramanujan made two deep conjectures about the coefficients of

$$
q \prod_{n=1}^{\infty}\left(1-q^{n}\right)^{24}=\sum_{n=1}^{\infty} \tau(n) q^{n} .
$$

The first was the multiplicativity of the coefficients: if $(m, n)=1$

$$
\tau(m n)=\tau(m) \tau(n)
$$


the second an estimate

$$
|\tau(n)| \leq d(n) n^{\frac{11}{2}},
$$

where $d(n)$ is the number of divisors of $n$. In particular,

$$
|\tau(p)| \leq p^{\frac{11}{2}}
$$

for primes $p$.

The first was proved by Mordell in 1917 and marked the beginning of Hecke's theory of Hecke operators. The second was proved by Deligne in 1974 and is one of the crowning achievements of twentieth century mathematics. $^{22}$

In his seminal 1965 paper 'On the estimation of Fourier coefficients of modular forms' Selberg formulated an analogue of Ramanujan's conjecture for nonholomorphic or Maass forms and showed that it is equivalent to the following statement about the first positive eigenvalue of the Laplacian (Selberg's eigenvalue conjecture ${ }^{23}$ ):

$$
\lambda_{1}(X(p)) \geq \frac{1}{4},
$$

where $X(p)=\mathbb{U} \backslash \Gamma(p)$, the quotient of the hyperbolic plane by the congruence subgroup

$$
\Gamma(p)=\left\{\gamma \in \mathrm{SL}_{2}(\mathbb{Z}): \gamma \equiv\left(\begin{array}{ll}
1 & 0 \\
0 & 1
\end{array}\right) \quad \bmod p\right\} .
$$

By the variational characterization of the first eigenvalue we have

$$
\lambda_{1}(X(p))=\inf _{\int_{X(p)} f d \mu=0} \frac{\int_{X(p)}|\nabla f|^{2} d \mu}{\int_{X(p)} f^{2} d \mu} .
$$

Using Weil's bound for Kloosterman sums (obtained as a consequence of his proof of the Riemann hypothesis for curves), Selberg proved the following celebrated result:

$$
\lambda_{1}(X(p)) \geq \frac{3}{16} .
$$

This result can be viewed as (implicitly) giving rise to the first family of expander graphs.

4.4. Expanders. Expanders are highly connected sparse graphs widely used in computer science. Clearly high connectivity is desirable in any communication network. The necessity of sparsity is perhaps best seen in the case of the network of neurons in the brain: since the axons have finite thickness their total length cannot exceed the quotient of the average volume of one's head and the area of axon's cross-section. In fact, this is the context in which expander

\footnotetext{
22'According to the author of the proof, Pierre Deligne, in order to present this proof, presupposing everything known by a beginning graduate student in mathematics one would need about two thousand pages of printed text. This theorem probably holds the record in modern mathematics for the ratio of the length of its proof to the length of its statement.' Y. Manin.

${ }^{23}$ See the article with eponymous title by P. Sarnak in the Notices for a tantalizing discussion.
}

graphs first implicitly appeared in the work of Barzdin and Kolmogorov in 1967.

There are several ways of making the intuitive notions of connectivity and sparsity precise; the simplest and most widely used is the following.

Given a subset of vertices, its boundary is the set of edges connecting the set to its complement. The expansion of a subset is a ratio of the size of a boundary to the size of a set. The expansion of a graph is a minimum over all expansion coefficients of its subsets.

The expansion coefficient captures the notion of being highly-connected, the bigger the expansion coefficient, the more highly-connected is the graph. Of course one can simply connect all the vertices but in this case the number of edges grows as the square of the number of vertices. The problem of constructing expanders is nontrivial because we put the second constraint: the graphs are to be sparse, i.e., the number of edges should grow linearly with the number of vertices. The simplest way to accomplish this is to demand that the graphs be regular, that is, each vertex has the same number of neighbors (say 3 ).

A family of $k$-regular graphs $\mathcal{G}_{n, k}$ forms a family of expanders if there is a fixed positive constant $c$, such that

$$
\liminf _{n \rightarrow \infty} c\left(\mathcal{G}_{n, d}\right) \geq c>0 .
$$

The expansion coefficient is a notion which is very easy to grasp but it is difficult to compute numerically or to estimate analytically, as the number of subsets grows exponentially with the number of vertices. The starting point of most current work on expanders is that the expansion coefficient has a spectral interpretation: ${ }^{24}$ to put it sonorously, if you hit a graph with a hammer, you can determine how highly-connected it is by listening to the bass note. In more technical terms, high connectivity is equivalent to establishing a spectral gap for an averaging (or Laplace) operator on the graph so that condition (31) has the following alternative expression:

$$
\liminf _{n \rightarrow \infty} \lambda_{1}\left(\Delta\left(\mathcal{G}_{n, k}\right)\right) \geq \mu>0,
$$

making apparent the connection with Selberg's celebrated $\frac{3}{16}$ Theorem (30).

In 1973 Pinsker observed that random regular graphs are expanders. In the same year Margulis gave the first explicit construction of expanders as Cayley graphs ${ }^{25}$ of $\mathrm{SL}_{3}\left(\mathbb{F}_{p}\right)$ using Kazhdan's property $\mathrm{T}$.

\footnotetext{
${ }^{24}$ The connection stems from the variational characterization of the first eigenvalue, expressed in (29).

${ }^{25}$ Given a finite group $G$ with a symmetric set of generators $S$, the Cayley graph $\mathcal{G}(G, S)$ is a graph which has elements of $G$ as vertices and which has an edge from $x$ to $y$ if and only if $x=\sigma y$ for some $\sigma \in S$. The Cayley graph of $\mathrm{PSL}_{2}\left(\mathbb{F}_{5}\right)$ with respect to standard generators is a buckyball, alluded to in the rendering of $\mathrm{O}$ on the conference poster (Figure 2).
} 
4.5. Superstrong approximation. The strong approximation for $\mathrm{SL}_{n}(\mathbb{Z})$, asserting that the reduction $\pi_{q}$ modulo $q$ is onto, is a consequence of the Chinese Remainder Theorem; its extension to arithmetic groups is far less elementary but well understood. If $S$ is a finite symmetric generating set of $\mathrm{SL}_{n}(\mathbb{Z})$, strong approximation is equivalent to the assertion that the Cayley graphs $\mathcal{G}\left(\mathrm{SL}_{n}(\mathbb{Z} / q \mathbb{Z}), \pi_{q}(S)\right)$ are connected. The quantification of this statement, asserting that they are in fact highly-connected, that is to say form a family of expanders, is what we mean by superstrong approximation. The proof of the expansion property for $\mathrm{SL}_{2}(\mathbb{Z})$ has its roots in Selberg's celebrated lower bound (30). The generalization of the expansion property to $G(\mathbb{Z})$ where $G$ is a semisimple matrix group defined over $\mathbb{Q}$ is also known thanks to developments towards the general Ramanujan conjectures that have been established; this expansion property is also referred to as property $\tau$ for congruence subgroups.

Let $\Gamma$ be a finitely generated subgroup of $\mathrm{GL}_{n}(\mathbb{Z})$ and let $G=\operatorname{Zcl}(\Gamma)$. The discussion of the previous paragraph applies if $\Gamma$ is of finite index in $G$. However if $\Gamma$ is thin, that is to say of infinite index in $G(\mathbb{Z})$, then $\operatorname{vol}(G(\mathbb{R}) \backslash \Gamma)=\infty$ and the techniques used to prove both of these properties do not apply. It is remarkable that under a suitable natural hypothesis, strong approximation continues to hold in this thin context, as proved by Matthews, Vaserstein, and Weisfeller in 1984. That the expansion property might continue to hold for thin groups was first suggested by Lubotzky in 1993; for $\mathrm{SL}_{2}(\mathbb{Z})$ the issue is neatly encapsulated in the following 1-2-3 question of his. For a prime $p \geq 5$ and $i=1,2,3$ let us define $S_{p}^{i}=\left\{\left(\begin{array}{ll}1 & i \\ 0 & 1\end{array}\right),\left(\begin{array}{ll}1 & 0 \\ i & 1\end{array}\right)\right\}$. Let $\mathcal{G}_{p}^{i}=\mathcal{G}\left(\mathrm{SL}_{2}(\mathbb{Z} / p \mathbb{Z}), S_{p}^{i}\right)$, a Cayley graph of $\mathrm{SL}_{2}(\mathbb{Z} / p \mathbb{Z})$ with respect to $S_{p}^{i}$. By Selberg's Theorem, $\mathcal{G}_{p}^{1}$ and $\mathcal{G}_{p}^{2}$ are families of expander graphs. However the group $\left\langle\left(\begin{array}{ll}1 & 3 \\ 0 & 1\end{array}\right),\left(\begin{array}{ll}1 & 0 \\ 3 & 1\end{array}\right)\right\rangle$ has infinite index and thus does not come under the purview of Selberg's Theorem.

Following the groundbreaking work of Helfgott (which builds crucially on the sum-product estimate in $\mathbb{F}_{p}$ discussed in section 3.4) Bourgain and Gamburd gave a complete answer to Lubotzky's question. The method introduced in 'Uniform expansion bounds for Cayley graphs of $\mathrm{SL}_{2}(\mathbb{Z} / p \mathbb{Z})^{\prime}$ and developed in a series of papers, became known as the 'Bourgain-Gamburd expansion machine;' thanks to a number of major developments by many people the general superstrong approximation for thin groups is now known. The state of the art is summarized in Thin Groups and Superstrong Approximation which contains an expanded version of most of the invited lectures from the eponymous MSRI 'Hot Topics' workshop, in the survey by Helfgott 'Growth in groups: Ideas and prospectives' in BAMS, and in the book by Tao Expansion in Finite Simple Groups of Lie Type.
4.6. On the spectral gap for finitely generated subgroups of $\mathrm{SU}(d)$. There is an Archimedean analogue of the expansion property, intimately related to the BanachRuziewicz problem discussed in section 4.2 , defined as follows.

For $k \geq 2$ let $g_{1}, \ldots, g_{k}$ be a finite set of elements in $G=$ $S U(d)(d \geq 2)$. We associate with them an averaging (or Hecke) operator $z_{g_{1}, \ldots, g_{k^{\prime}}}$ taking $L^{2}(S U(d))$ into $L^{2}(S U(d))$ :

$$
z_{g_{1}, \ldots, g_{k}} f(x)=\sum_{j=1}^{k} f\left(g_{j}(x)\right)+f\left(g_{j}^{-1}(x)\right) .
$$

We denote by $\operatorname{supp}(z)$ the set $\left\{g_{1}, \ldots, g_{k}, g_{1}^{-1}, \ldots, g_{k}^{-1}\right\}$ and by $\Gamma_{z}$ the group generated by $\operatorname{supp}(z)$. It is clear that $z_{g_{1}, \ldots, g_{k}}$ is self-adjoint and that the constant function is an eigenfunction of $z$ with eigenvalue $\lambda_{0}(z)=2 k$. Let $\lambda_{1}\left(z_{g_{1}, \ldots, g_{k}}\right)$ denote the supremum of the eigenvalues of $z$ on the orthogonal complement of the constant functions in $L^{2}(S U(d))$. We say that $z$ has a spectral gap if $\lambda_{1}\left(z_{g_{1}, \ldots, g_{k}}\right)<2 k$. It is common to, alternatively, refer to the situation described above, by asserting that the spectral gap property holds for $\Gamma_{z}$.

It is easy to see that an affirmative solution of BanachRuziewicz follows from the existence of $z$ in $\mathrm{SU}(2)$ having a spectral gap. In their 1986 paper, referenced at the end of section 4.2, Lubotzky, Philips and Sarnak posed a question of whether generic in measure $z$ in $\mathrm{SU}(2)$ has a spectral gap.

In 2008 Bourgain and Gamburd proved (Theorem 9 below) the spectral gap property for $z$ in $\mathrm{SU}(2)$ satisfying the noncommutative diophantine property (NDP) - in particular for free subgroups generated by elements with algebraic entries.

The definition of noncommutative diophantine property ${ }^{26}$ introduced in the paper 'Spectra of elements in the group ring of $\mathrm{SU}(2)$ ' by Gamburd, Jakobson and Sarnak is as follows. We say that $z_{g_{1}, \ldots, g_{k}}$ satisfies NDP if there is $D=D\left(g_{1}, \ldots, g_{k}\right)>0$ (the diophantine constant of $z$ ) such that for any $m \geq 1$ and a word $W_{m}$ in $g_{1}, \ldots, g_{k}$ of length $m$ with $W_{m} \neq \pm e$ (where $e$ denotes the identity in $\mathrm{SU}(2)$ ) $\left\|W_{m} \pm e\right\| \geq D^{-m}$.

Theorem 9. Let $g_{1}, \ldots, g_{k}$ be a set of elements in $\mathrm{SU}(2)$ generating a free group and satisfying NDP (in particular, elements with algebraic entries). ${ }^{27}$ Then $z_{g_{1}, \ldots, g_{k}}$ has a spectral gap.

\footnotetext{
${ }^{26}$ Recall that $\theta \in \mathbb{R}$ is called diophantine if there are positive constants $C_{1}, C_{2}$ s.t. for all $(k, l) \in \mathbb{Z}^{2}$ with $k \neq 0$ we have $|k \theta-l| \geq c_{1} k^{-c_{2}}$. Equivalently, letting $g=e^{2 \pi \theta} \in \mathrm{SO}(2)$ we may re-express this condition as follows: $\left|g^{k}-1\right| \geq$ $c_{1}^{\prime} k^{-c_{2}^{\prime}}$. A classical result asserts that diophantine numbers are generic in measure in $\mathbb{R}$. Given diophantine $\theta_{1}, \ldots, \theta_{k}$ and $g_{1}=e^{2 \pi \theta_{1}}, \ldots, g_{k}=e^{2 \pi \theta_{k}}$ in $\mathrm{SO}(2)$, for any word $W$ in $g_{1}, \ldots, g_{k}$ of length $m$ we have $\left|W_{m}-1\right| \geq c_{1} m^{-c_{2}}$ for some $c_{1}, c_{2}$. In the case of $\mathrm{SO}(3)$, given $g_{1}, \ldots, g_{k}$ generating a free subgroup, a pigeonhole argument shows that for any $m \geq 1$ there is always a word $W$ in $g_{1}, g_{1}^{-1}, \ldots, g_{k}, g_{k}^{-1}$ of length at most $m$ such that $\left\|W_{m}-e\right\| \leq 10(2 k-1)^{-\frac{m}{6}}$, so the exponential behavior in the definition below is the appropriate one.

${ }^{27}$ It was established in [GJS] that elements with algebraic entries satisfy NDP.
} 
Regarding the proof, let me just note that in the adaption of the 'expansion machine' to this Archimedean setting, the crucial role is played by the following strengthening of Theorem 7.

Theorem 10. Given $0<\delta<1$ and $\kappa>0$ there exists $\varepsilon_{0}>0$ and $\varepsilon_{1}>0$ such that if $\delta>0$ is sufficiently small and $A \subset$ $[1,2]$ is a discrete set consisting of $\delta$-separated points, satisfying $|A|=\delta^{-\sigma}$ and

$$
|A \cap I|<\rho^{\kappa}|A|
$$

whenever I is a size $\rho$ interval with $\delta<\rho<\delta^{\varepsilon_{0}}$, then

$$
N(A+A, \delta)+N(A \cdot A, \delta)>\delta^{-\varepsilon_{1}}|A| .
$$

Theorem 9 is of importance in quantum computing. In the context of quantum computation elements of a threedimensional rotation group are viewed as 'quantum gates' and a set of elements generating a dense subgroup is called 'computationally universal' (since any element of a rotation group can be approximated by some word in the generating set to an arbitrary precision). A set of elements is called 'efficiently universal' if any element can be approximated by a word of length which is logarithmic with respect to the inverse of the chosen precision (this is the best possible). A consequence of Theorem 9 is that computationally universal sets with algebraic entries are efficiently universal.

Another application is related to the theory of quasicrystals. Generalizing Penrose's two-dimensional aperiodic tiling, John Conway and Charles Radin constructed a self-similar (hierarchical) tiling of a three-dimensional space with a single prototile, such that the tiles occur in an infinite number of different orientations in the tiling. The tile is a prism, which when scaled up by 2 is subdivided into eight copies of itself ('daughter tiles'). If one iterates this same subdivision procedure over and over, one creates in the limit the desired tiling of three-dimensional space by prisms. Conway and Radin showed that the orientations of tiles in the tiling are uniformly distributed and posed the question of how fast this convergence to uniform distribution takes place. This question reduces to the study of the spectral gap for the averaging operator associated with eight rotations giving orientations of daughter tiles. A consequence of Theorem 9 is that this convergence takes place exponentially fast.

\section{Coda}

The essense of mathematics lies precisely in its freedom.

$$
\text { Georg Cantor }
$$

Already history has in a sense ceased to exist, i.e. there is no such thing as a history of our own times which could be

A major open question is whether $z$ generic in measure in $\mathrm{SU}(2)$ satisfies NDP. The best known result in this direction is due to Kaloshin and Rodnianski: for almost every pair $(A, B)$ in $\mathrm{SU}(2) \times \mathrm{SU}(2)$ there is a constant $D>0$ s.t. for any $n$ and any word $W_{m}\left\|W_{m}(A, B) \pm e\right\| \geq D^{-m^{2}}$. universally accepted, and the exact sciences are endangered as soon as military necessity ceases to keep people up to the mark. Hitler can say that the Jews started the war, and if he survives, that will become official history. He can't say that two and two are five, because for the purposes of, say, ballistics they have to make four.

George Orwell, letter to N. Wilmett, 18 May 1944

Freedom is the freedom to say that two plus two make four. If that is granted, all else follows.

George Orwell, Nineteen Eighty-Four, 1949

The difficulties of explaining Bourgain's work to a broad mathematical audience turned out to be quite substantiali ${ }^{28}$ omitting 'mathematical' from the appellation renders them nearly insurmountable.

Ian Stewart begins his admirable book The Problems of Mathematics (Oxford University Press, 1987) with an interview with a mathematician conducted by Seamus Android on behalf of the proverbial man-in-the-street ${ }^{29}$ invoked in Hilbert's celebrated 1900 address Problems of Mathematics, referenced at the beginning of section 2 .

\footnotetext{
Mathematician: It's one of the most important discoveries of the last decade!

Android: Can you explain it in words ordinary mortals can understand?

Mathematician: Look, buster, if ordinary mortals could understand it, you would not need mathematicians to do the job for you, right? You can't get a feeling for what's going on without understanding the technical details. How can I talk about manifolds without mentioning that the theorem only works if the manifolds are finite dimensional paracompact Hausdorff with empty boundary?

Android: Lie a bit.

Mathematician: Oh, but I could not do that!

Android: Why not? Everybody else does.
}

Perhaps the most troubling omen of our times is an assault on the very basic notions of logic and truth, in their most elemental Aristotilean sense, including, in particular, the law of the excluded middle. Our discipline stands as a mighty fortress against this assault; and I, for one, believe we should not be overly defensive about our reluctance to lie a bit just because everybody else does.

$$
* * *
$$

Of all escapes from reality, mathematics is the most successful ever. It is a fantasy that becomes all the more addictive because it works back to improve the same reality we are trying to evade. All other escapes - sex, drugs, hobbies, whatever are ephemeral by comparison. The mathematician's feeling of

\footnotetext{
28 'There is a continuing need to lead new generations along the thorny path which has no shortcuts. The Ancients said there is no royal road in mathematics. But the vanguard is leaving the great mass of pilgrims further and further behind, the procession is ever more strung out, and the leaders are finding themselves alone far out ahead.' H. Steinhaus

${ }^{29}$ The proverbial meaning is a function of street's location in space-time cultural continuum: it is exceedingly unlikely, I reckon, that Aristotle's remark in Nicomachean Ethics, IX that 'without friends none would care to live, though having all other things besides' should necessarily be construed as endorsement of Facebook.
} 
triumph, as he forces the world to obey the laws his imagination has freely created, feeds on its own success. The world is permanently changed by the workings of his mind, and the certainty that his creations will endure renews his confidence as no other pursuit.

Gian-Carlo Rota, 'The Lost Cafe', 1987

The one who writes a poem writes it above all because verse writing is an extraordinary accelerator of conscience, of thinking, of comprehending the universe. Having experienced this acceleration once, one is no longer capable of abandoning the chance to repeat this experience; one falls into dependency on this process, the way others fall into dependency on drugs or on alcohol. One who finds himself in this sort of dependency on language is, I guess, what they call a poet.

Joseph Brodsky, 'Nobel Lecture', 1987

To paraphrase W. H. Auden (writing In Memory of W. B. Yeats),

[Mathematics] ${ }^{30}$ makes nothing happen: it survives

In the valley of its making, where executives

Would never want to tamper.

In attempting to explain the significance of Bourgain's remarkable and remarkably useful results to a proverbial human-online, one may invoke their applications in mathematical physics, computer science and cryptography, which are of immense practical importance in contemporary life, making, in particular, online communication possible. Their subtlety, beauty and depth appear to be much harder to convey in 'plain English.' Here and now, perhaps, we must remind ourselves that the humanonline, while attached to a digital device (built by von Neumann) is still human and sound-bite/tweet thus: while dealing with entities seemingly fake/unreal (e.g. the real line), Bourgain's singular adventures in the labyrinth of the continuum represent a magnificent and transcendent achievement of the human spirit.

I met Jean in September 2005, six months after my daughter (who drew the pictures for this essay) was born, while visiting IAS for the program 'Lie Groups, Representations and Discrete Mathematics' led by Alex Lubotzky. I do not remember the precise date but do remember the hour: it was between 2 and $3 \mathrm{am}$. After changing my daughter's diapers I could not sleep, went to Simonyi Hall and ran into Jean walking to the Library. It was in this discombobulated state that I was free of fear to speak to him. By dawn, the problem which had been resisting my protracted attack for a decade was vanquished in Jean's office. ${ }^{31}$

\footnotetext{
${ }^{30}$ Save e.g. hydrogen bomb and computer.

${ }^{31}$ Jean had the following daily routine. He would arrive at the dining hall for lunch within 5 minutes of its closing and, while descending the stairs, would look for whom to join for the meal (the relevance of the person was determined primarily by their expertise in the problem Jean was currently working on). After lunch and before sunset the door of his office would be half-open. After getting a bottle of red wine (typically Medoc), Jean would have dinner around 9 pm, followed by a double espresso (typically in Small World Coffee), return to
}

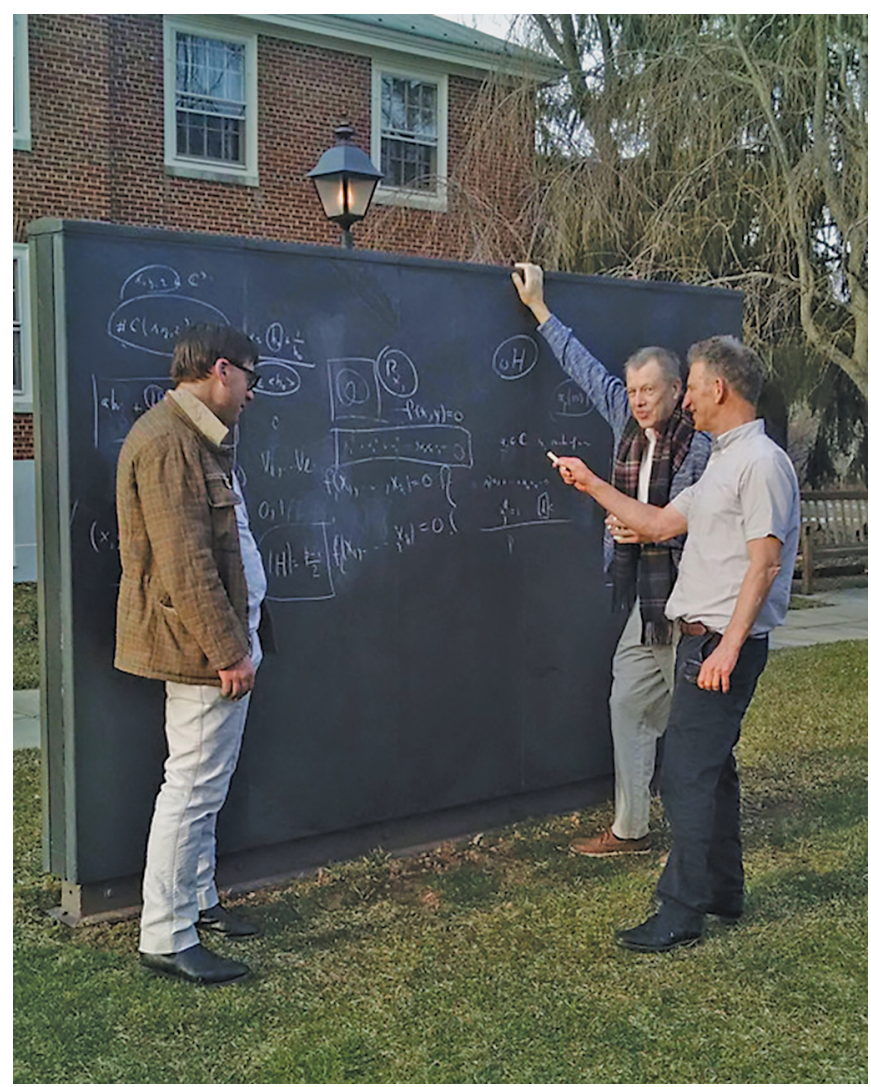

Figure 10. Jean Bourgain, Peter Sarnak, Alex Gamburd.

During this happiest year of my life, in 2005-2006, I stayed on the Lane named after Hermann Weyl who was of the view that 'Mathematics is not the rigid and uninspiring schematism which the layman is so apt to see in it; on the contrary, we stand in mathematics precisely at that point of limitation and freedom which is the essence of man himself.'

During my second visit to IAS, in 2007-2008, as a von Neumann Fellow participating in the 'Arithmetic Combinatorics' Program led by Jean Bourgain and Van Vu, I stayed on the Lane named after Erwin Panofsky. His magnificent essay The History of Art as a Humanistic Discipline, based on The Spencer Trask Princeton University Lectures for 1937-38, commences thus:

Nine days before his death Immanuel Kant was visited by his
physician. Old, ill, and nearly blind, he rose from his chair
and stood trembling with weakness and muttering unintelli-
gible words. Finally his faithful companion realized that he
would not sit down until the visitor has taken a seat. This he
did, and Kant then permitted himself to be helped to his chair,

the office, call his wife and son, and then go for a brisk walk, encircling Einstein Drive about five times. Between midnight and sunrise his office door would typically be closed. His hand-written notes (like that of Mozart's and unlike Beethoven's) are virtually free of corrections, in part, because during his dinner and walk he would think about what would be set to paper upon his return to the office. 
and, after regaining some of his strength, said, 'Das Gefühl für Humanität hat mich noch nicht verlassen' - 'The sense of humanity has not yet left me.' The two men were moved almost to tears. For, though the word Humanität had come, in the eighteenth century, to mean little more than politeness or civility, it had, for Kant, a much deeper significance, which the circumstances of the moment served to emphasize: man's proud and tragic consciousness of self-approved and self-imposed principles, contrasting with his utter subjection to illness, decay and all that is implied in the word 'mortality'.

Towards the end of the essay, Panofsky thus (pre-) echoes Orwell: 'If the anthropocratic civilization of the Renaissance is headed, as it seems to be, for a Middle Ages in reverse-a satanocracy as opposed to the mediaeval theocracy-not only the humanities but also natural sciences, as we know them, will disappear, and nothing will be left but what serves the dictates of the sub-human.'

During my third, short visit, I stayed on von Neumann Drive (the only other 'Drive' at IAS is named after Einstein). The similarities between von Neumann and Baron Bourgain are subtle and striking.

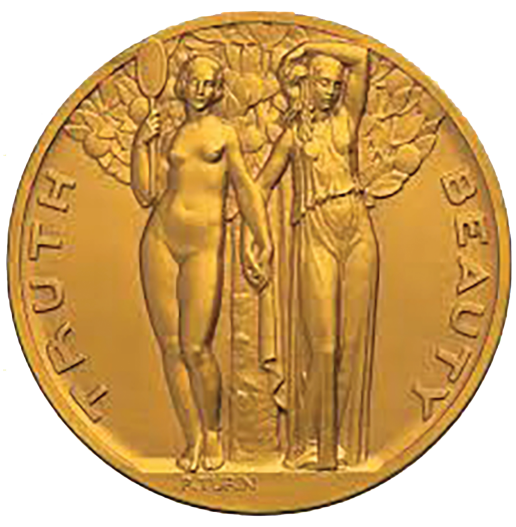

Figure 11. The IAS Seal.

The IAS (where Jean did most of the work described in this essay) official seal is imprinted on the Analysis and Beyond conference poster. In a circular format, the quiet elegant and classical Art Deco composition depicts two graceful young ladies, one clothed and one otherwise, standing on opposite sides of a leafy tree that appears to bear abundant fruit. Their poses are complementary, one looking out toward the spectator, the other looking down, avoiding eye contact. The figures are named in large sans serif letters, TRUTH to the left, BEAUTY on the right. Truth holds a mirror that overlaps the circular frame to reflect reality.

Underlaying the design of the seal is the evident allusion to the famous final couplet of 'Ode on a Grecian Urn': 'Beauty is truth, truth beauty,-that is all Ye know on earth, and all ye need to know by John Keats, who was of the view that 'the excellence of every art is its intensity, capable of making all disagreebles evaporate from their being in close relationship with Beauty and Truth.'

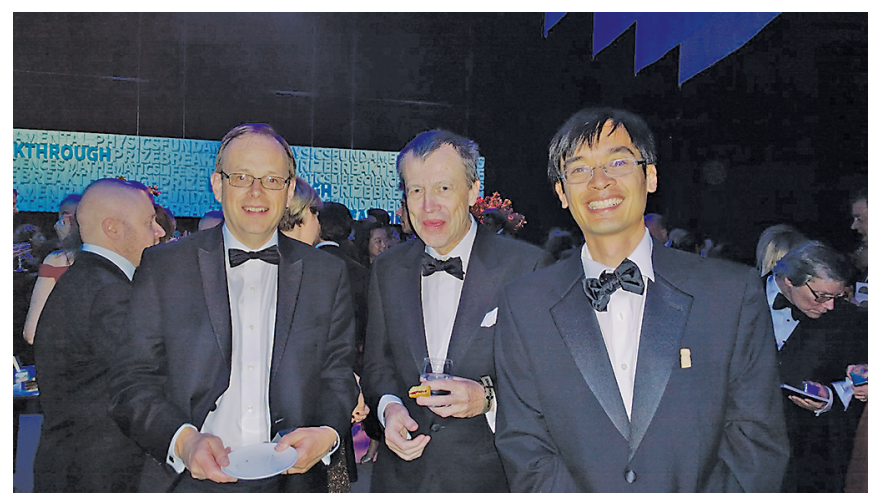

Figure 12. Richard Taylor, Jean Bourgain, Terence Tao.

Having attempted in this essay a snapshot of the excellence of Bourgain's art, let me conclude by giving a glimpse of his intensity by quoting from the interview upon receiving the 2017 Breakthrough Prize in Mathematical Sciences:

If you have a question which is generally perceived as unapproachable, it is often that you do not even quite know where you have to look to get a solution. From that point of view, we are rather like Fourier, ${ }^{32}$ stranded in the desert, hopelessly lost. At the moment you get this insight, all of a sudden you escape the desert and things open up for you. Then we feel very excited. These are the best moments. They make up for all the suffering with absolutely no progress worth it.

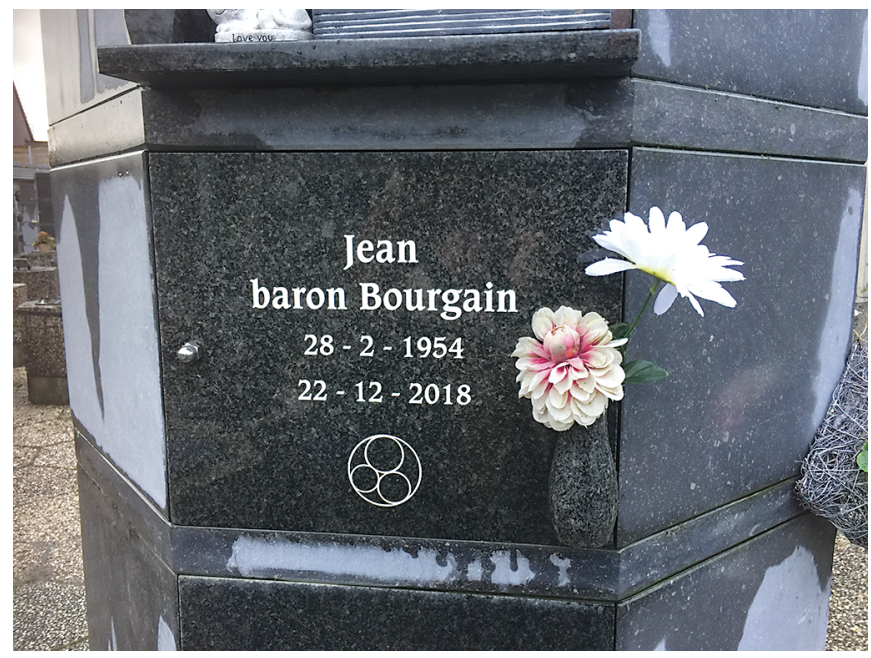

Figure 13.

\footnotetext{
${ }^{32}$ Jean-Baptiste Joseph Fourier was a member of General Bonaparte's expedition to Egypt (1798-1801), important enough for the First Consul to make him, in 1802, the Prefect of the département at Grenoble, a position which he held until Emperor Napoleon's fall.
} 
ACKNOWLEDGMENTS. The work on this essay commenced in late December 2016, prompted by Frank Morgan's solicitation of ad honorem Bourgain contributions for the Notices; it was completed two years later, 20 December 2018, while the author had the honor and privilege of enjoying the hospitality of the Hausdorff Institute in Bonn, 200 kilometers and two days separated from the place and time of Bourgain's death, in Bonheiden (Belgium) on 22 December 2018.

The author was supported, in part, by NSF award DMS-1603715.

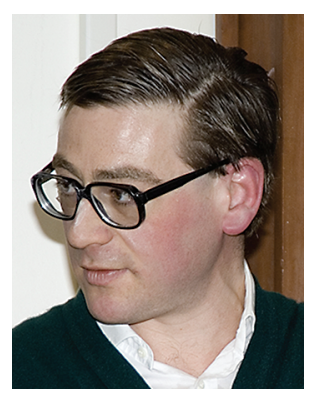

Alexander

Gamburd

Credits

Opening image and Figures 1, 2, and 11 are courtesy of the Institute for Advanced Study.

Figures 3 and 9 and photo of Alex Gamburd are courtesy of C. J. Mozzochi.

Figure 4 is courtesy of Svetlana Jitomirskaya.

Figure 5 is courtesy of Kenneth Falconer.

Figures 6 and 8 are courtesy of Anna Gamburd.

Figure 7 is courtesy of Erik Huss ${ }^{\circ}$ Royal Swedish Academy of Sciences.

Figure 10 is courtesy of Enrico Bombieri.

Figure 12 is courtesy of Terence Tao.

Figure 13 is courtesy of Claire Bourgain.

\section{JOIN THE AMS...}

or renew your membership today

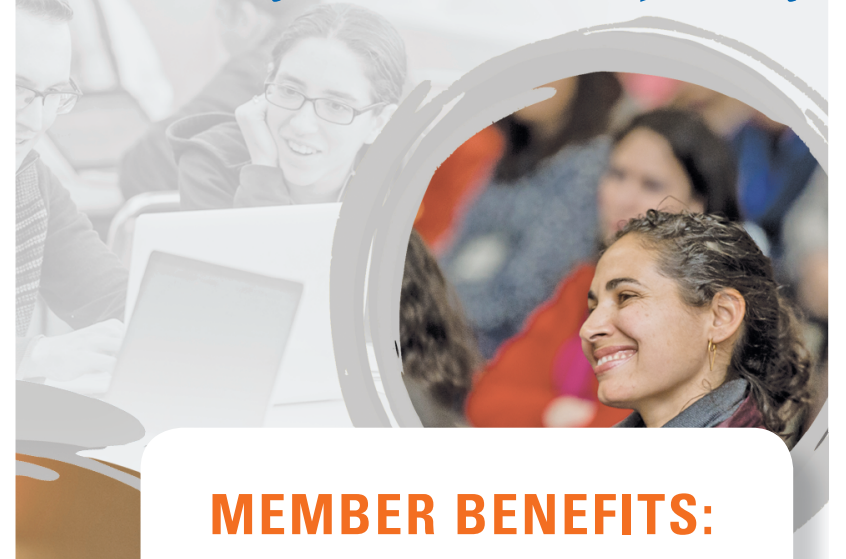

- Individual AMS members receive free standard shipping on orders delivered to addresses in the United States (including Puerto Rico) and Canada

- Discounts on AMS publications including MAA Press books

- Subscriptions to Notices and Bulletin

- Discounted registration for world-class meetings and conferences

- Access to online AMS Member Directory

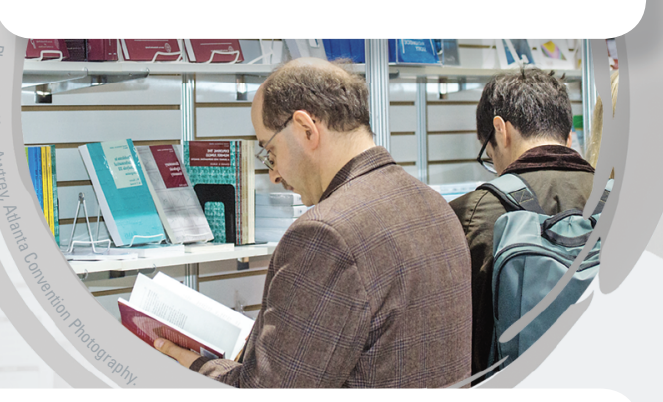

Learn more and join online at www.ams.org/membership.

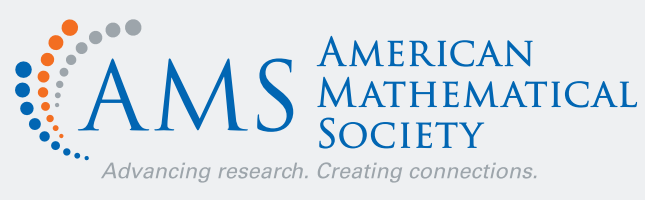

